\title{
Measurement of Action Potential-Induced Presynaptic Calcium Domains at a Cultured Neuromuscular Junction
}

\author{
David A. DiGregorio, Arthur Peskoff, and Julio L. Vergara \\ Department of Physiology, University of California at Los Angeles School of Medicine, Los Angeles, California 90095
}

\begin{abstract}
Spatially localized $\mathrm{Ca}^{2+}$ domains are thought to play a key role in action potential (AP)-evoked neurotransmitter release at fast synapses. We used a stage-scan confocal spot-detection method and the low-affinity $\mathrm{Ca}^{2+}$ indicator Oregon Green 488 BAPTA-5N to study the spatiotemporal profile of presynaptic AP-induced $\mathrm{Ca}^{2+}$ domains. Families of scanned AP-induced fluorescence transients were detected from spot locations separated by $200-300 \mathrm{~nm}$, within the vicinity of $\mathrm{Ca}^{2+}$ entry sites. Typically, the largest transient in a particular scan peaked within $\sim 1 \mathrm{msec}$ and decayed with rapid $\left(\tau_{1}\right.$ of $\left.1.7 \mathrm{msec}\right)$ and slow components ( $\tau_{2}$ of $16 \mathrm{msec}, \tau_{3}$ of $78 \mathrm{msec}$ ). As the spot was incrementally displaced, transients progressively exhibited a slowing in their time-to-peak and a loss of the fast decay component. Three-dimensional graphs of fluorescence versus time and spot displacement revealed the presence of AP-
\end{abstract}

induced fluorescence domains that dissipated within $\sim 7 \mathrm{msec}$. The size of fluorescence domains were estimated from the full-width at half-maximum of gaussian fits to isochronal $\Delta F / F$ plots and ranged from 0.6 to $3.0 \mu \mathrm{m}$, with a mean \pm SD of $1.6 \pm$ $0.6 \mu \mathrm{m}$. Model simulations of a localized $\mathrm{Ca}^{2+}$ entry site predicted the major features of the fluorescence transients and suggested that, within $\sim 1$ msec of the initiation of the $\mathrm{Ca}^{2+}$ current, both the fluorescence domain and the underlying $\mathrm{Ca}^{2+}$ domain do not extend significantly beyond the site of entry. Consistent with this prediction, the intracellular addition of EGTA (up to $2 \mathrm{~mm}$ ) accelerated the decay of the measured transients but did not affect the domain size.

Key words: neuromuscular junction; presynaptic calcium; calcium transients; calcium indicators; calcium microdomains; synaptic transmission
It is well known that neurotransmitter release at fast synapses is triggered by $\mathrm{Ca}^{2+}$ entry into the presynaptic terminal after action potential (AP) invasion (Katz, 1969; Llinas et al., 1981; Augustine et al., 1987; Borst and Sakmann, 1996; Sabatini and Regehr, 1996; Yazejian et al., 1997). This $\mathrm{Ca}^{2+}$ entry into nerve terminals is mediated through voltage-activated $\mathrm{Ca}^{2+}$ channels thought to be clustered near sites of vesicle fusion or active zones (Heuser and Reese, 1981; Pumplin et al., 1981; Roberts et al., 1990). It is thought that the time course of neurotransmitter release is set by the transient nature of localized $\left[\mathrm{Ca}^{2+}\right]$ changes (Barrett and Stevens, 1972; Zucker and Stockbridge, 1983; Stockbridge and Ross, 1984; Yamada and Zucker, 1992). Consequently, direct measurement and characterization of the spatiotemporal profiles of these AP-induced $\mathrm{Ca}^{2+}$ domains is of crucial importance for understanding fast $\mathrm{Ca}^{2+}$-dependent synaptic transmission.

Most of what is known about the temporal and spatial characteristics of $\mathrm{Ca}^{2+}$ domains is based on mathematical model simulations (Chad and Eckert, 1984; Fogelson and Zucker, 1985; Simon and Llinas, 1985; Parnas et al., 1989; Yamada and Zucker, 1992; Cooper et al., 1996; Bertram et al., 1999). Unfortunately, these models lack a direct comparison with experimental measurements of $\mathrm{Ca}^{2+}$ domains. This is, in part, because of technical limitations in the optical methods commonly used to measure

Received May 10, 1999; revised June 29, 1999; accepted July 7, 1999.

This work was supported by National Institutes of Health Grant AR25201 (to J.L.V.), Medical Scientist Training Program Grant GM 08042, and National Institutes of Health individual Fellowship NS10197 (to D.A.D). We thank Viet Tran and Mike Panian for the preparation of the cell cultures. We also thank Jeremy Dittman, Albert Kim, Sally Krasne, Fernando Marengo, Jonathan Monck, and Valentin Naegerl for helpful discussions and comments on this manuscript.

Correspondence should be addressed to Dr. Julio Vergara, Department of Physiology, University of California at Los Angeles School of Medicine, 10833 LeConte Avenue, 53-263 Center for Health Sciences, Los Angeles, CA 90095-1751.

Copyright (C) 1999 Society for Neuroscience $0270-6474 / 99 / 197846-14 \$ 05.00 / 0$ intracellular $\left[\mathrm{Ca}^{2+}\right]$ (Mason, 1993). Scanned confocal fluorescence microscopy provides adequate spatial resolution (Wilson, 1990) for the identification of submicrometer $\mathrm{Ca}^{2+}$-dependent fluorescence domains in response to long (>50 msec) depolarizations (Tucker and Fettiplace, 1995; Issa and Hudspeth, 1996; Hall et al., 1997). However, the investigation of presynaptic APinduced $\mathrm{Ca}^{2+}$ domains requires the use of low-affinity $\mathrm{Ca}^{2+}$ indicators and fast sampling rates (Escobar et al., 1994; DiGregorio and Vergara, 1997). Evidence of $\mathrm{Ca}^{2+}$ domains has been obtained using the luminescent protein aequorin at the squid giant synapse (Sugimori et al., 1994).

The cultured Xenopus neuromuscular junction (NMJ) preparation is well suited for the study of fast presynaptic $\mathrm{Ca}^{2+}$ domains associated with neurotransmitter release. In this preparation, the spontaneously forming synaptic contacts have been shown to exhibit functional and structural similarities to those of the mature NMJ (Katz, 1969; Weldon and Cohen, 1979; Buchanan et al., 1989; Yazejian et al., 1997). Structural studies using fluorescence staining methods in the cultured preparation have demonstrated that both synaptic vesicles and postsynaptic receptors colocalize in discrete regions of nerve-muscle contact (Cohen et al., 1987). Consistent with this finding, we found that rapid $\mathrm{Ca}^{2+}$ transients could be detected in discrete localized regions of the presynaptic terminal (DiGregorio and Vergara, 1997).

Here, we combined a high spatial resolution stage-scan device with the confocal spot-detection system (Escobar et al., 1994; DiGregorio and Vergara, 1997) to characterize the temporal and spatial profile of AP-induced $\mathrm{Ca}^{2+}$ domains. We found that the $\mathrm{Ca}^{2+}$-dependent fluorescence domains develop and dissipate within milliseconds and that their full-width at half-maximum (FWHM) reflects the size of the underlying $\mathrm{Ca}^{2+}$ entry site. 


\section{MATERIALS AND METHODS}

Cell culture and electrophysiology. Xenopus nerve-muscle cocultures were prepared from dissociated neural tube-myotome tissue obtained from embryos at stages 20-22, as described previously (Yazejian et al., 1997). The dissociated cells were plated onto glass coverslips and allowed to grow for $18-36 \mathrm{hr}\left(\right.$ at $22-24^{\circ} \mathrm{C}$ ) before experimental use. Neuronal cell bodies were patch-clamped to elicit soma APs and to dialyze the presynaptic terminal with a pipette solution containing Oregon Green 488 BAPTA-5N (OGB-5N) ( $K_{\mathrm{d}}$ of $32 \mu \mathrm{M}$; Molecular Probes, Eugene, OR) (DiGregorio and Vergara, 1997). APs were recorded using an Axopatch 1D amplifier (Axon Instruments, Foster City, CA) filtered at $5 \mathrm{kHz}$ and acquired at $17-55 \mathrm{kHz}$ with a Digidata $1200 \mathrm{~A}$ data acquisition system operating under software control (pClamp 7.0; Axon Instruments). All experiments were performed at room temperature $\left(19-21^{\circ} \mathrm{C}\right)$.

Solutions. Neuronal patch electrodes (5-10 $\mathrm{M} \Omega$ resistance) were backfilled with a solution of the following composition (in $\mathrm{mm}$ ): 85 $\mathrm{K}$-aspartate, $20 \mathrm{KCl}, 403$ - $(\mathrm{N}$-morpholino) propane sulfonic acid (MOPS), $0.5 \mathrm{MgCl}_{2}, 0.01-2$ EGTA, 2 ATP-Mg, and 0.5 GTP-Na $2, \mathrm{pH}$ 7.0. Unless otherwise indicated, the pipette solution also contained 600 $\mu \mathrm{M}$ OGB-5N. Normal frog Ringer's solution (NFR), consisting of 114 $\mathrm{NaCl}, 2.5 \mathrm{KCl}, 10 \mathrm{MOPS}, 1.8 \mathrm{CaCl}_{2}$, and $10 \mathrm{D}$-glucose, $\mathrm{pH} 7.0$, was used to bathe the cultures during recording periods. D-Tubocurarine $(20 \mu \mathrm{M}$; Sigma, St. Louis, MO) was added to the NFR to block synaptically evoked myocyte contraction.

Cell imaging and spot illumination-detection. The optical system consisted of an inverted microscope (Diaphot; Nikon, Tokyo, Japan) equipped for epi-illumination with an argon laser (5 W; Spectra-Physics, Mountain View, CA) or a $100 \mathrm{~W}$ mercury lamp, a $100 \times$ oil immersion objective (Plan Fluor 100, 1.3 NA; Nikon) objective, a cooled CCD camera (MCD-600; Spectra Source, Agoura Hills, CA), and a photodiode. Fluorescence measurements used a $460-490 \mathrm{~nm}$ excitation filter, a dichroic mirror (505DRLP), and a 515 long-pass emission filter (all filters were obtained from Omega Optical, Brattleboro, VT). Fluorescence and phase-contrast images, used to document recording sites along the nerve terminal, were acquired with the cooled CCD camera.

AP-induced fluorescence transients were recorded from nerve terminals using a confocal spot-detection method similar to that described previously (Escobar et al., 1994, 1997; DiGregorio and Vergara, 1997). Briefly, light from a laser-illuminated source pinhole $(5 \mu \mathrm{m}$ in diameter) was projected through the epi-illumination port of the microscope (with a $10 \times$ objective) and focused to a "spot" on the specimen with the high NA $100 \times$ objective. The fluorescence image of the illumination spot was centered on the square active area $\left(0.04 \mathrm{~mm}^{2}\right)$ of a photodiode (HR008; United Detector Technology, Hawthorne, CA) mounted at the image plane on the lateral port of the microscope. The diode current was amplified using the capacitor-feedback mode of an Axopatch 200B amplifier (Axon Instruments), filtered at corner frequencies of $1-3 \mathrm{kHz}$ using an eight-pole Bessel filter (Frequency Devices, Haverhill, MA), and digitized at $20-55 \mathrm{kHz}$ using the Digidata $1200 \mathrm{~A}$.

To evaluate the dimensions of the fluorescence illumination-detection volume, it was necessary to estimate the diameter of the illumination spot and the depth discrimination of the detector. The laser-illuminated pinhole was focused onto a thin homogenous layer ( $\sim 4-\mu \mathrm{m}$-thick) of 100 $\mu \mathrm{M}$ fluorescein (Sigma) solution and imaged with the CCD camera (Fig. $1 A)$. The pixel intensity along a horizontal line bisecting the fluorescence spot is plotted as a function of spatial location (Fig. $1 \mathrm{~B}$, double arrow). The diameter of the focused spot can be approximated by the FWHM of the intensity profile of the illumination system (Wilson, 1990; Pawley, 1995), which in our case is $0.68 \mu \mathrm{m}$ (Fig. 1B, arrows). A small diameter such as this limits the axial dimension of the illumination volume to an FWHM of $<2 \mu \mathrm{m}$ (Hiraoka et al., 1990; Castleman, 1996). The horizontal scale bar in Figure $1 B$ represents the size of the detection aperture at the image plane $(2 \mu \mathrm{m})$, which is determined by the active area of the photodiode. Although a detector of this dimension does not improve the lateral resolution of the optical detection system beyond that set by the FWHM of the illumination spot, it can enhance the axial depth discrimination to a minimum of $1.3 \mu \mathrm{m}$ (Wilson, 1990).

Stage-scan spot detection. Scanned spot experiments were performed by laterally displacing the preparation with respect to the optical axis of the illumination-detection system. Electrode micromanipulators and cell cultures were mounted on a custom-made microscope stage equipped with manual $x-y$ micrometers and an additional high-resolution $(100 \mathrm{~nm})$ stepper motor (UTS20PP.1; Newport, Irvine, CA) to allow high precision movements along one axis $(x)$. The motor was computer controlled using
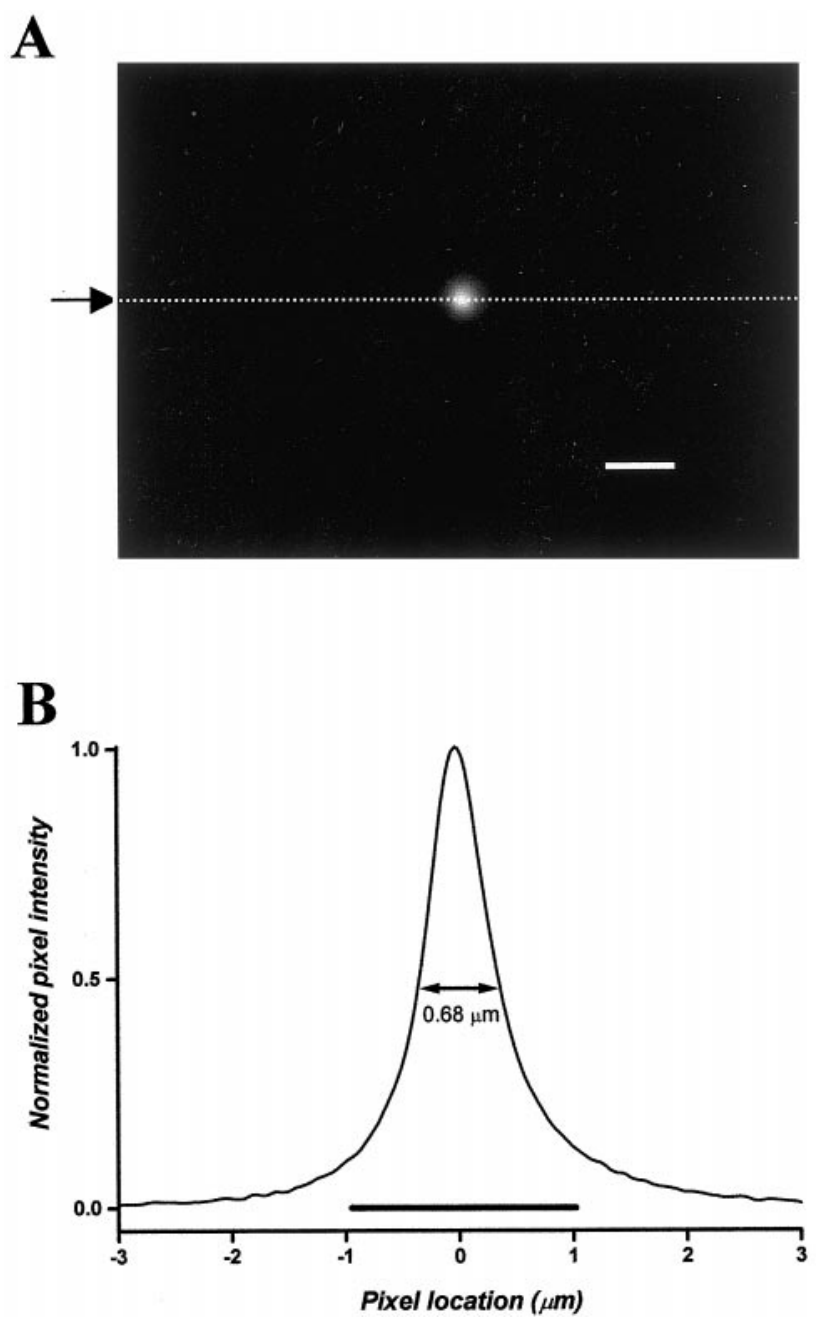

Figure 1. Lateral dimensions of the illumination spot. $A$, CCD image $(228 \times 312$ pixels $)$ of the argon laser-illuminated pinhole focused in a thin layer $(\sim 4 \mu \mathrm{m})$ of solution containing $100 \mu \mathrm{m}$ fluorescein. Scale bar, $2 \mu \mathrm{m}$. $B$, Intensity profile along a single row of pixels (arrow in $A$ ) centered on the illumination spot. The double arrow indicates the FWHM of the intensity line profile. The black bar represents the dimension of the photodiode in the image plane.

proprietary hardware and Windows95 software (Prairie Technologies, Waunakee, WI).

For calibration scans, various sizes of fluorescent beads (Molecular Probes) were fixed to a glass coverslip and bathed in distilled water. The spatially dependent fluorescence intensity was assayed in $100 \mathrm{~nm}$ increments by time-averaging the fluorescence for 100-150 msec at each spot location.

Measurement and analysis of fluorescence transients. The $\mathrm{Ca}^{2+}$ indicator OGB-5N was allowed to diffuse from the cell body to the nerve terminal for at least 20 min subsequent to establishing a whole-cell configuration and before optical recordings. In scanned experiments, consecutive AP-induced fluorescence transients were recorded from adjacent spot locations separated by 200 or $300 \mathrm{~nm}$ in the $x$ direction. The lateral spot displacements were made before each AP delivered at intervals of 5-10 sec. However, for multiple recordings at a single location, APs were delivered every 20-30 sec to minimize photodynamic damage (DiGregorio and Vergara, 1997). The total illumination period per acquisition was set to $200-400 \mathrm{msec}$ using an electronic shutter (Newport). APs were delivered 100-200 msec after shutter opening.

$\Delta F / F$ traces were calculated according to the definition

$$
\frac{\Delta F}{F}(t)=\frac{F(t)-F_{\text {rest }}}{F_{\text {rest }}}
$$




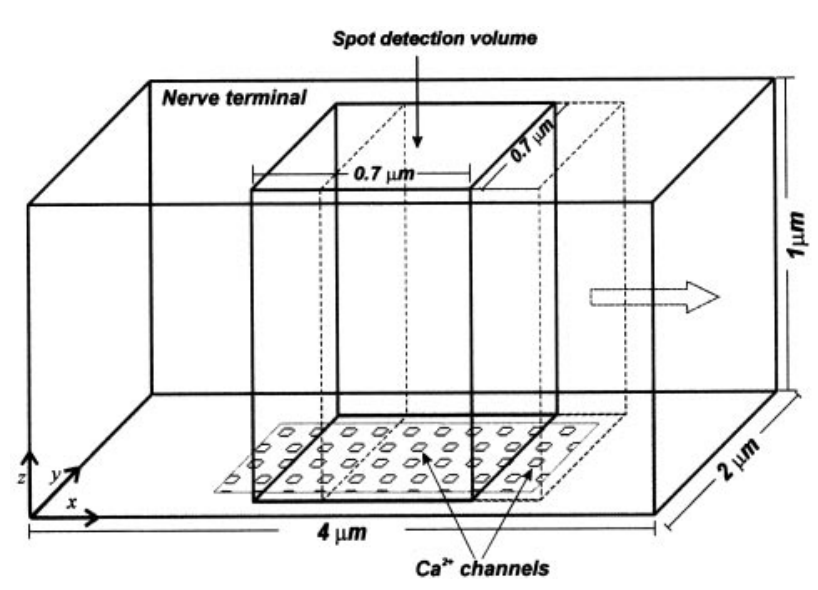

Figure 2. Schematic diagram illustrating the major features of the diffusion-reaction model. $\mathrm{Ca}^{2+}$ ions were allowed to enter the nerve terminal (dimensions as indicated) through channels located within a discrete region of the membrane ( $\mathrm{Ca}$ entry site). The illumination-detection region was modeled as a parallelepiped (Spot detection volume) of the dimensions indicated. To simulate the spatial dependence of scanned fluorescence transients, the detection volume was displaced in the direction of the $x$-axis (large arrow). The $y$ - and $z$-axes are indicated for reference.

where $F_{\text {rest }}$ is the resting fluorescence before stimulation, and $F(t)$ is the time-dependent fluorescence transient. In cases in which the resting fluorescence did not exhibit a flat baseline (e.g., because of bleaching of the dye), the $F_{\text {rest }}$ term in the $\Delta F / F$ calculation was determined from a single exponential fit to the fluorescence trace before AP stimulation. The exponential function was then extended for the length of the record and subtracted from the entire trace. The resulting experimental trace was finally scaled by $F_{\text {rest }}$ to produce the $\Delta F / F$ record. For the purposes of signal averaging or to make kinetic comparisons between laterally displaced transients, the jitter in the AP initiation time was corrected by time shifting both the voltage and fluorescence traces such that the time of peak of soma APs coincided.

The decay phase of scaled and normalized fluorescence transients was fitted, using a least-square algorithm (Origin 5.0; Microcal, Northampton, MA), to a triple exponential decay function according to:

$$
\frac{\Delta F}{F}(t)=A_{1} \mathrm{e}^{-t / \tau_{1}}+A_{2} \mathrm{e}^{-t / \tau_{2}}+A_{3} \mathrm{e}^{-t / \tau_{3}}
$$

where $A_{1}-A_{3}$ and $\tau_{1}-\tau_{3}$ are the fitted relative amplitudes and time constants, respectively.

Fluorescence variance traces were calculated from individual fluorescence transients $\left(\frac{\Delta F}{F}(t)\right)_{i}$ and their mean $\left(\frac{\overline{\Delta F}}{F}(t)\right)$, according to the following equation:

$$
\sigma_{F}^{2}(t)=\frac{1}{N-1} \sum_{i=1}^{N}\left(\left(\frac{\Delta F}{F}(t)\right)_{i}-\frac{\overline{\Delta F}}{F}(t)\right)^{2}
$$

$N$ is either the number of traces recorded at a single site or the number of recording locations in a scan experiment. The calculations for singlesite fluorescence variance analysis were similar to those for $\mathrm{Na}^{+}$current nonstationary fluctuation analysis (Sigworth, 1980).

Statistical significance of the difference between two data sets was determined by obtaining $p$ values using a two-tailed Student's $t$ test.

Mathematical model of diffusion within a presynaptic terminal. We constructed a mathematical model to compute the spatiotemporal distribution of the free $\left[\mathrm{Ca}^{2+}\right],[\mathrm{Ca}](x, y, z, t)$, and the concentration of $\mathrm{Ca}^{2+}$ bound to buffers, $\left[\mathrm{Ca} B_{\mathrm{i}}\right](x, y, z, t)$, after $\mathrm{Ca}^{2+}$ entry through channels (Fig. 2, $\mathrm{Ca}^{2+}$ channels) organized in a discrete region of the presynaptic terminal membrane (" $\mathrm{Ca}^{2+}$ entry site"). The dimensions of the nerve terminal were set to $4 \times 2 \times 1 \mu \mathrm{m}$ (Fig. 2), and those of the $\mathrm{Ca}^{2+}$ entry site were set as indicated for each simulation. The interior of the terminal was assumed to be a homogeneous medium in which $\mathrm{Ca}^{2+}$ diff uses and reacts with immobile and mobile buffers. Specifically, we included one immobile endogenous buffer, $B_{1}$, and two mobile exogenous buffers $B_{2}$ (EGTA) and $B_{3}$ (OGB-5N), using experimental values for their association $\left(k_{i}^{+}\right)$and dissociation $\left(k_{i}^{-}\right)$rate constants. The diffusion-reaction equations for $\mathrm{Ca}^{2+}$ ions and the three $\mathrm{Ca}^{2+}$ buffer complexes $(\mathrm{CaB}, i=$ 1,2,3) are, respectively:

$$
\begin{gathered}
\frac{\partial[C a]}{\partial t}=D_{C a}\left(\frac{\partial^{2}[C a]}{\partial x^{2}}+\frac{\partial^{2}[C a]}{\partial y^{2}}+\frac{\partial^{2}[C a]}{\partial z^{2}}\right)-\sum_{i=1}^{3} \frac{\partial\left[C a B_{i}\right]}{\partial t} \\
+S(x, y, z, t) \\
\frac{\partial\left[C a B_{i}\right]}{\partial t}=D_{i}\left(\frac{\partial^{2}\left[C a B_{i}\right]}{\partial x^{2}}+\frac{\partial^{2}\left[C a B_{i}\right]}{\partial y^{2}}+\frac{\partial^{2}\left[C a B_{i}\right]}{\partial z^{2}}\right) \\
+k_{i}^{+}[C a]\left(\left[B_{i}\right]^{\text {total }}-\left[C a B_{i}\right]\right)-k_{i}^{-}\left[C a B_{i}\right]
\end{gathered}
$$

where $D_{\mathrm{Ca}}, D_{2}$, and $D_{3}$ are the diffusion coefficients of $\mathrm{Ca}^{2+}$ and exogenous buffers 2 and 3 , respectively. $D_{1}$ is set to zero to account for an immobile buffer.

Numerical integration of the model equations was performed using an explicit finite-difference (Euler) algorithm with a fixed time step and an elementary integration volume (voxel) with dimensions $\Delta x, \Delta y$, and $\Delta z$ set to $0.1 \mu \mathrm{m}$. In this computation, the concentration at each grid point at a given time is computed in terms of the concentration at that grid point and the six adjacent grid points at the previous time point. The computed concentration at a grid point in the interior of the terminal represents the average concentration in the $0.1 \times 0.1 \times 0.1 \mu \mathrm{m}$ cubic voxel centered on the grid point. The computed concentration at a grid point on the membrane surface represents the average in a truncated voxel $(0.1 \times$ $0.1 \times 0.05 \mu \mathrm{m})$ extending away from the membrane located at $\mathrm{z}=0$, halfway to the adjacent grid point at $z=0.1 \mu \mathrm{m}$.

$S(x, y, z, t)$ is a $\mathrm{Ca}^{2+}$ source function that was set to zero everywhere, except for specified discrete elements $\left(x_{\mathfrak{i}}, y_{\mathrm{i}}\right)$ in the $x-y$ plane in which $\mathrm{Ca}^{2+}$ channels are located, according to the equation:

$$
S\left(x_{i}, y_{i}, 0, t\right)=\frac{I_{C a}(t)}{2 F \Delta x \Delta y \frac{\Delta z}{2}}
$$

In Equation 5, $I_{\mathrm{Ca}}$ is the $\mathrm{Ca}^{2+}$ current entering each volume element at the membrane boundary and $F$ is Faraday's constant. The time course (in milliseconds) of $I_{\mathrm{Ca}}$ was calculated according to the gaussian function:

$I_{C a}(t)=A e^{-\left(t-t_{\text {peak }}\right)^{2} / 2 \cdot \sigma^{2}}$, where $t_{\text {peak }}$ represents the time of the peak $\mathrm{Ca}^{2+}$

entry and was set to $1 \mathrm{msec}$, and $\sigma$ was set to 0.35 . The time course of this function (half-width, $\sim 0.4 \mathrm{msec}$ ) resembles that of experimentally measured nerve terminal $\mathrm{Ca}^{2+}$ currents using AP command waveforms (Yazejian et al., 1997). $A$ is set to $0.25 \mathrm{pA}$, which approximates the single-channel current of a $1.4 \mathrm{pS}$ channel (Umemiya and Berger, 1995; Church and Stanley, 1996) assuming a maximal driving force of $175 \mathrm{mV}$.

Other model parameters were selected from literature values and our own experimental measurements. $D_{\mathrm{Ca}}$ was set to $200 \mu \mathrm{m}^{2} / \mathrm{sec}$, similar to that measured in cytosolic extract from Xenopus oocytes (Allbritton et al., 1992). The nerve terminal contained an immobile buffer with kinetic properties identical to those estimated in adrenal chromaffin cells $\left(k_{1}^{+}=\right.$ $\left.1.0 \times 10^{8} \mathrm{M}^{-1} \mathrm{sec}^{-1}, k_{1}^{-}=10,000 \mathrm{sec}^{-1}\right)(\mathrm{Xu}$ et al., 1997). The total concentration of the immobile buffer $\left(\left[B_{1}\right]^{\text {total }}\right)$ was set to $2 \mathrm{~mm}$. The nerve terminal also contained a $\mathrm{Ca}^{2+}$ indicator $\left(B_{2}\right)$ at the concentration used in the experiments $\left(\left[B_{2}\right]^{\text {total }}=600 \mu \mathrm{M}\right)$. Its rate constants were set to those measured for OGB-5N ( $k_{2}^{+}=1.7 \times 10^{8} \mathrm{M}^{-1} \mathrm{sec}^{-1}$ and $k_{2}^{-}=$ $5600 \mathrm{sec}^{-1}$ (DiGregorio et al., 1998) using flash photolysis of 1-(4,5dimethoxy-2-nitrophenyl) EDTA (DM-nitrophen) (Escobar et al., 1997). Various amounts of the mobile exogenous buffer EGTA $\left(B_{3}\right)$ were also included in the model to match experimental conditions, and its kinetic rate constants were set to $k_{3}^{+}=6.0 \times 10^{6} \mathrm{M}^{-1} \mathrm{sec}^{-1}$ and $k_{3}^{-}=0.78 \mathrm{sec}^{-1}$. These values were obtained from in vitro flash photolysis experiments performed at $\mathrm{pH} 7.0$ (identical to that of the pipette solution) at $20^{\circ} \mathrm{C}$. Both $k_{3}^{+}$and $k_{3}^{-}$were estimated by fitting a flash photolysis mathematical 
model (Escobar et al., 1997) to OGB-5N fluorescence transients obtained at various [EGTA] and flash intensities (D. A. DiGregorio, J. J. Marengo, and J. L. Vergara, unpublished observations). The value of $k_{3}^{+}$is $\sim 4$ times faster than that measured by Smith et al. (1984) at pH 7.0 and approximately twice as fast as that estimated by Naraghi (1997) at $\mathrm{pH}$ 7.2. The value $K_{\mathrm{d}}(0.13 \mu \mathrm{M})$ is slightly less than the $0.2 \mu \mathrm{M}$ of Smith et al. (1984) and the $0.18 \mu \mathrm{M}$ of Naraghi (1997). The diffusion coefficients of OGB-5N $\left(D_{2}\right)$ and EGTA $\left(D_{3}\right)$ were set to $100 \mu \mathrm{m}^{2} / \mathrm{sec}$ which is comparable with reported values (Pape et al., 1995; Gabso et al., 1997).

We computed the $\Delta F / F$ in each volume element (voxel) of the nerve terminal according to following equation:

$$
\frac{\Delta F}{F}(t)=\frac{\left[\mathrm{CaB}_{2}\right](t)-\left[\mathrm{CaB}_{2}\right](0)}{\frac{B_{2}^{\text {total }}}{R-1}+\left[\mathrm{CaB}_{2}\right](0)}
$$

where $R$ is the ratio of the maximal to minimal fluorescence for OGB-5N $\left(F_{\max } / F_{\min }=26\right)\left(\right.$ DiGregorio and Vergara, 1997) and $\left[\mathrm{CaB}_{2}\right](0)$ is the equilibrium concentration of the $\mathrm{Ca}^{2+}$-dye complex at a resting $\left[\mathrm{Ca}^{2+}\right]$ of $0.1 \mu \mathrm{M}$. To compare model simulations with experimental traces, we calculated the average $\Delta F / F$ over the entire spot-detection volume (Fig. 2). Unless otherwise indicated, the $x-y$-dimensions of the spot-detection volume were set to match the FWHM $(0.7 \mu \mathrm{m})$ of the illumination spot image (Fig. 1), and the depth was set to $1 \mu \mathrm{m}$ (the $z$-dimension of the whole terminal). Furthermore, to compare with fluorescence traces recorded at different locations, the spot-detection volume (Fig. 2, dotted rectangular parallelepiped) was displaced in the $x$ direction.

Model computations were implemented in Fortran-90 (Fortran Power Station 4.0; Microsoft, Redmond, WA) on a $400 \mathrm{MHz}$ Pentium II-based personal computer.

\section{RESULTS}

\section{Spatial dependence of AP-induced fluorescence transients}

Using phase-contrast microscopy, we identified nerve terminals in contact with muscle cells for the detection of AP-induced fluorescence transients. By moving the preparation with respect to the optical axis of the microscope, the illumination-detection spot was positioned at random along the contact region of the nerve terminal and repositioned until a rapid fluorescence transient was detected in response to AP stimulation (DiGregorio and Vergara, 1997). From that location, the nerve terminal was scanned with the illumination-detection spot at high resolution to obtain a family of fluorescence transients. Figure 3 illustrates a typical experiment in which a neuronal cell body was whole-cell patched-clamped and presynaptic fluorescence transients were recorded. Figure $3 A$, left, is a CCD image of a nerve terminal loaded with $600 \mu \mathrm{M}$ OGB-5N acquired using global epifluorescence illumination. In Figure $3 A$, to the right is a diagram of the same nerve terminal at a slightly higher magnification to illustrate the relative size of the illumination spot (circle) and the length of the scan within the nerve terminal (double arrow). A current pulse injection into the neuronal cell body elicited the AP shown in Figure $3 B$ (black trace), which propagated to the nerve terminal in which a fluorescence trace was recorded. The bottom traces in Figure $3 B$ are a set of single AP-elicited OGB-5N fluorescence transients obtained in consecutive trials when displacing the spot location in increments of $300 \mathrm{~nm}$. The red trace is the fluorescence transient with the largest peak $\Delta F / F$ and was recorded from a spot location midway through this particular scan (Fig. $3 A$, circle). As the spot was displaced from the site of maximal fluorescence (red through magenta), several features of the transients progressively changed: there was a reduction in the peak amplitude, a slowing of the time-to-peak, and a loss of the rapid decay phase. However, after $\sim 10 \mathrm{msec}$, all the traces exhibit a common slow decay time component. To better visualize the spatial dependence of the
$\mathbf{A}$

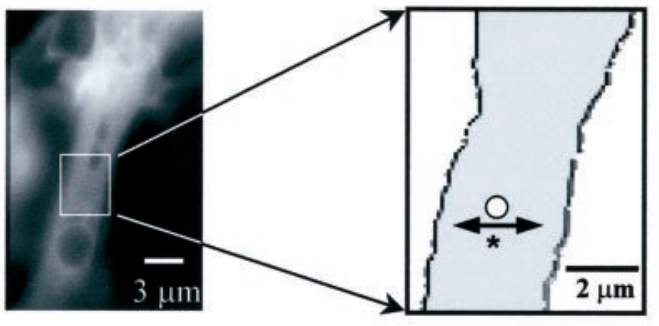

B
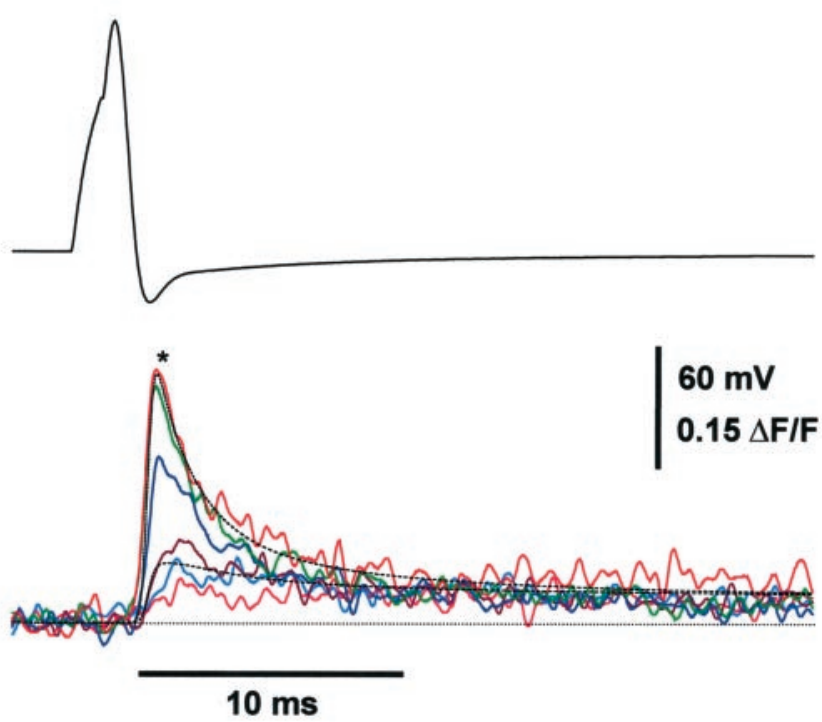

C

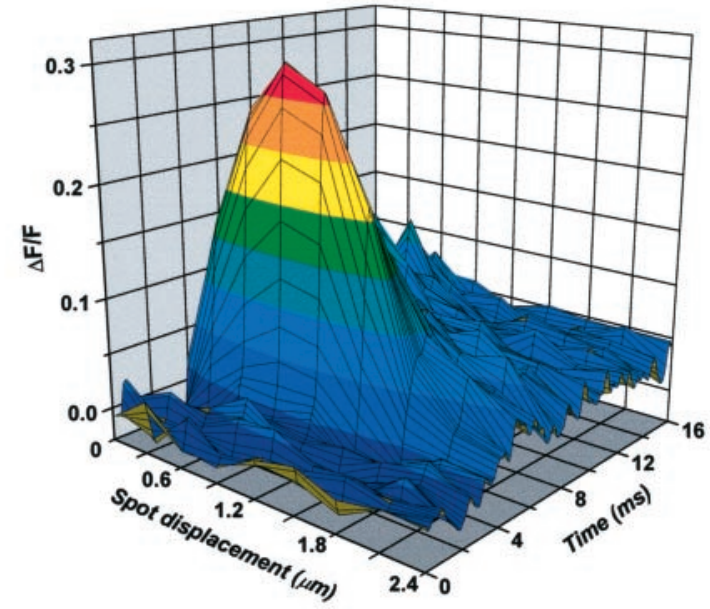

Figure 3. Spatial dependence of AP-induced presynaptic fluorescence transients. $A, \mathrm{CCD}$ image of a nerve terminal loaded with $600 \mu \mathrm{M}$ OGB-5N. Right, Camera lucida diagram of the same nerve terminal at higher magnification illustrating the size of the illumination spot (open circle) relative to the nerve terminal. The arrow indicates the direction of the spot displacement, and the asterisk indicates the location in which the largest transient was acquired. $B$, A family of single AP-induced OGB-5N fluorescence transients (bottom traces) recorded consecutively at various spot locations in $0.3 \mu \mathrm{m}$ steps along the horizontal line (double arrow in $A)$. The black trace corresponds to the average of the six neuronal APs that elicited the fluorescence records. EGTA $(50 \mu \mathrm{M})$ was included in the pipette solution. Fluorescence traces were filtered at $1 \mathrm{kHz}$. Two model simulation traces are superimposed on the experimental records (black dotted traces). $C$, Three-dimensional plot obtained by offsetting all the fluorescence traces in the scan according to their relative spot-detection location. Color bands are increments of $0.05 \Delta F / F$. 

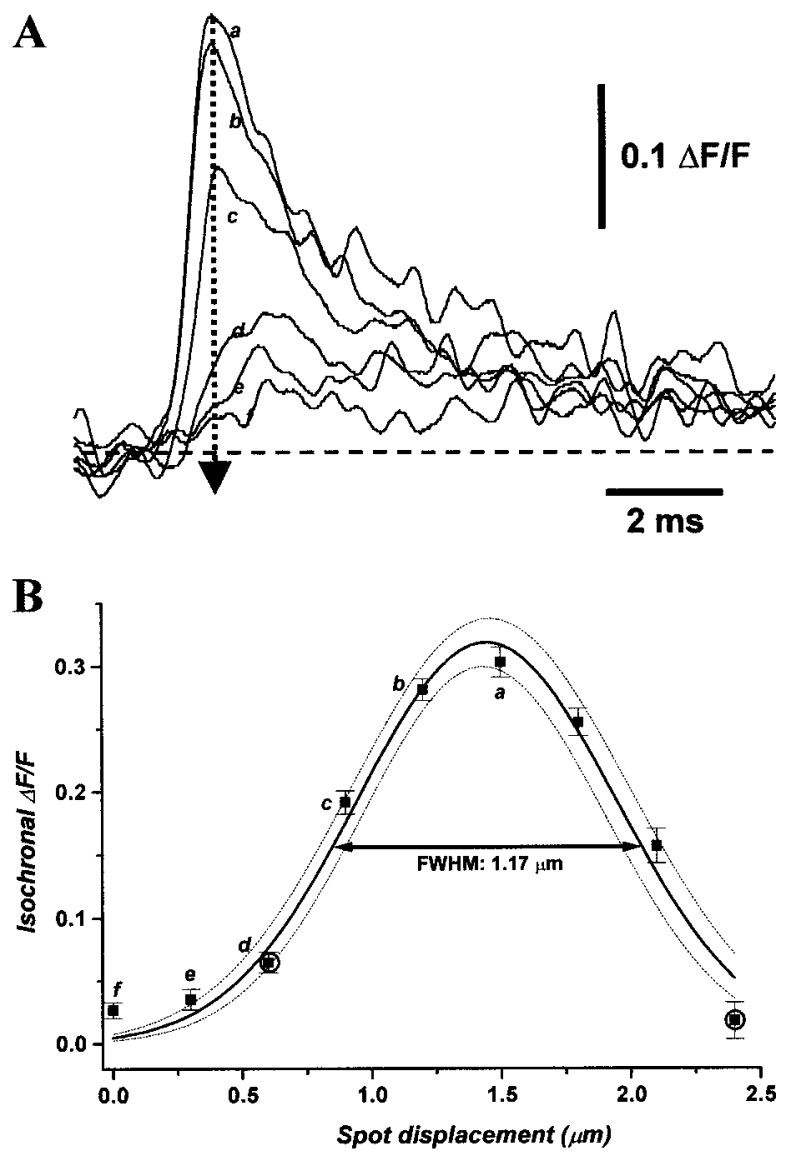

Figure 4. Size of fluorescence domain as determined from the FWHM of an isochronal $\Delta F / F$ plot. $A$, Fluorescence transients from the same experiment in Figure 3, shown in an expanded time scale to illustrate the isochronal time point (arrow). The traces were labeled $a-f$ to indicate successive $0.3 \mu \mathrm{m}$ displacements. $B$, Isochronal $\Delta F / F$ values plotted as a function of spot displacement. The letters correspond to the trace in $A$ from which the isochronal values were obtained. The thick curve is the gaussian fit to data points between, and including those, enclosed in circles. Error bars represent the SD, calculated from $10 \mathrm{msec}$ of baseline noise, for each individual fluorescence transient. The dashed curves represent the gaussian fits when $2 \cdot S D$ was added to (top) or subtracted from (bottom) the plotted data points.

OGB-5N fluorescence transients, each record from this scan was plotted in a three-dimensional graph (Fig. $3 C$ ), as a function of the spot displacement. In this plot, fluorescence transients detected over a range of $\sim 2 \mu \mathrm{m}$ demonstrate the presence a $\mathrm{Ca}^{2+}$ dependent fluorescence "domain" that dissipates within $\sim 10$ msec.

\section{Estimation of domain size using isochronal $\Delta \boldsymbol{F} / \boldsymbol{F}$}

To quantify the size of fluorescence domains, we estimated the FWHM of isochronal $\Delta F / F$ versus spot displacement plots using fluorescence values acquired early after AP stimulation. Figure 4 illustrates this analysis as applied to the domain in Figure 3. Figure $4 A$ shows that, at the time point (dotted arrow) when the largest fluorescence transient (trace $a$ ) peaked ( $1 \mathrm{msec}$ after its initiation), the differences between the amplitudes of scanned transients were maximal. In addition, we know that neurotransmitter release is initiated in this preparation within $\sim 1 \mathrm{msec}$ of AP invasion (DiGregorio and Vergara, 1997; Yazejian et al., 1997). Therefore, we selected this time as a reasonable isochronal point at which the domain size would be most prominent and relevant. Each ordinate value in Figure $4 B$ corresponds to the magnitude of each fluorescence trace at the isochronal point. It can be observed that, by increasing the spot distance from the site of maximal $\Delta F / F$, the latency in the onset of transients was increased, thereby exacerbating the space-dependent reduction in the isochronal $\Delta F / F$ values.

In Figure $4 B$, the abscissa denotes the distance between spot locations at which individual transients were recorded. Zero displacement corresponds to the spot location from which the scan was initiated. The ordinate of each point was obtained by averaging the $\Delta F / F$ values within $\pm 150 \mu$ sec of the isochronal point. The FWHM of the spatial dependence in the isochronal $\Delta F / F$ plot was obtained by fitting the data to a gaussian function (Fig. 4B, thick trace) using a least-square algorithm. The outermost data points of the fitted region (Fig. $4 B$, black circles) were selected to constrain the gaussian within the boundaries of an apparent single domain. The FWHM of the gaussian function was calculated to be $1.17 \mu \mathrm{m}$ from the best fit of the width parameter $\sigma(\mathrm{FWHM}=2 \sigma \sqrt{2 \ln 2}=2.35 \sigma)$. The error in the FWHM calculation introduced by the noise of the data was estimated from fits of the isochronal $\Delta F / F$ values adjusted by adding and subtracting $2 \cdot S D$ for each data point. The FWHM of the fit when $2 \cdot S D$ was added to each data point (Fig. $4 B$, top dotted trace) was $1.25 \mu \mathrm{m}$, whereas the FWHM of the fit when 2 - $S D$ was subtracted from each data point (Fig. $4 B$, bottom dotted trace) was $1.10 \mu \mathrm{m}$. This analysis provides a $95 \%$ confidence limit of $\pm 0.08 \mu \mathrm{m}$.

FWHM values estimated from scanned fluorescence transients in eight other nerve terminals under similar experimental conditions (10-50 $\mu \mathrm{M}$ EGTA) ranged from 0.75 to $3.0 \mu \mathrm{m}$, with an average of $1.5 \mu \mathrm{m}$ and a SD of $0.7 \mu \mathrm{m}(n=9)$ (see Table 2). Unfortunately, not all domains were derived from scanned fluorescence transients with signal-to-noise ratios $(\mathrm{S} / \mathrm{N})$ as large as those shown in Figure 3. We therefore estimated the error in the FWHM calculated from a fit to an isochronal $\Delta F / F$ plot obtained from fluorescence transients with a more typical S/N (Fig. 5A) and thus assessed our ability to discriminate between FWHM values. In Figure $5 A$, the red trace corresponds to the fluorescence transient with the largest peak $\Delta F / F$, and, as the spot was displaced in $200 \mathrm{~nm}$ steps (green through orange), there was a reduction in the peak amplitude similar to that shown in Figure $3 A$. When the transients are plotted versus spot displacement, the resulting fluorescence domain appeared narrower (Fig. $5 B$ ) than that of Figure $3 C$. The isochronal $\Delta F / F$ plot in Figure $5 C$ was obtained following the same procedure as described in Figure $4 B$. The gaussian fit to the isochronal $\Delta F / F$ values yielded an FWHM of $0.86 \mu \mathrm{m}$. The $95 \%$ confidence limit, estimated from fits of the data $\pm 2 \cdot S D$, was $\pm 0.18 \mu \mathrm{m}$. It can be observed that this noise-dependent error is larger than the $0.08 \mu \mathrm{m}$ calculated for the domain in Figure 4. Nevertheless, because the FWHM of both domains ( 0.86 and $1.17 \mu \mathrm{m}$, respectively) differ by $0.31 \mu \mathrm{m}$, more than the sum of both confidence intervals $(0.26 \mu \mathrm{m})$, we conclude that they are of significantly different size. The tendency of isochronal $\Delta F / F$ values to depart from baseline, as observed for spot locations to the left of the boundary data point (Fig. $5 C$, left cyan circle), suggests the presence of a neighboring domain that was excluded from the size determination.

In addition to the pairwise comparison of domains sizes presented above, it was useful to assess the average contribution of the noise to the estimate of the mean FWHM over the population of domains. To evaluate this error contribution, we first calculated the average SD from the resting fluorescence (before 
A
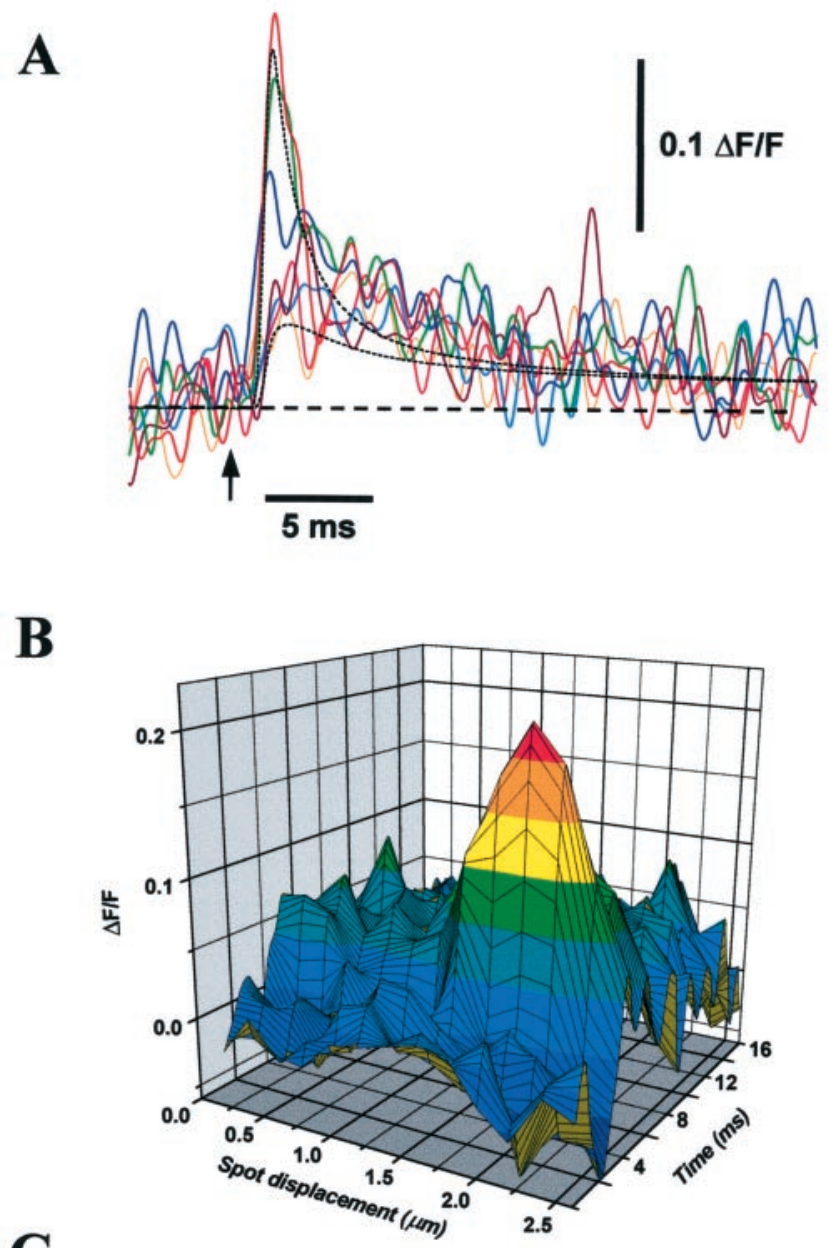

C

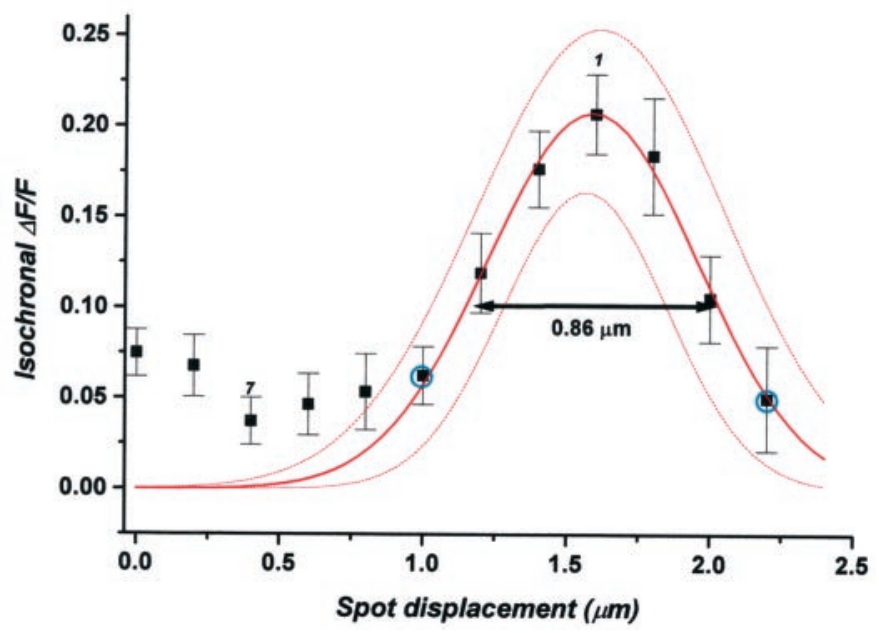

Figure 5. Small AP-induced fluorescence domain. A, Individual fluorescence transients recorded from a presynaptic terminal in the presence of $50 \mu \mathrm{M}$ EGTA. The traces were filtered at $1 \mathrm{kHz}$ and smoothed using a 2 $\mathrm{kHz}$ fast Fourier transform filter. The arrow indicates the time when the soma APs peaked. $B$, Three-dimensional plot of all the transients of this particular scan. $C$, Isochronal $\Delta F / F$ plot of data obtained from transients in $B$ (averaged within $\pm 225 \mu \mathrm{sec}$ of the isochronal point). The gaussian curve (solid red), yielding an FWHM (arrow) of $0.86 \mu \mathrm{m}$, was fitted to the data points between, and including those, enclosed in cyan circles. The data points labeled 1 and 7 correspond to the red through orange traces in $A$, respectively. Error bars and confidence limits (red dashed curves) were calculated as in Figure 4.
AP stimulation) of the experimental records for a particular domain. We then obtained the ratio of this value to that of the largest isochronal $\Delta F / F$ in that domain. This ratio provides a scaled uncertainty for every domain, which, when averaged over the population of domains, yields a scaled error value of $\pm 13 \%$. Thus, assuming a gaussian with the mean FWHM of the population $(1.5 \mu \mathrm{m})$, we estimate an average noise-dependent $95 \%$ confidence limit $( \pm 2 \cdot S D)$ of $\pm 0.33 \mu \mathrm{m}$. This is fourfold less than the $1.4 \mu \mathrm{m}$ confidence interval of the mean FWHM (see Table 2 ), suggesting that the heterogeneity in domain sizes cannot be explained by the noise in the fluorescence transients.

\section{Spatial calibration of the spot illumination-detection system}

To determine the relationship between measured FWHM values and size of the underlying fluorescence domains, we scanned calibration beads of known diameters in $100 \mathrm{~nm}$ steps using the spot-detection method. A typical example of the spatially dependent fluorescence profile obtained when a $0.46 \mu \mathrm{m}$ bead was scanned is illustrated in Figure $6 A$. The FWHM of this particular bead, estimated from the linearly interpolated fluorescence plot, was $0.70 \mu \mathrm{m}$.

To complete the size calibration, we performed identical experiments on beads of diameters ranging from 0.11 to $2.1 \mu \mathrm{m}$. Figure $6 B$ is a plot of pooled data comparing the FWHM of the measured intensity profiles versus the actual bead diameter. It can be observed that, for a bead diameter of $2.1 \mu \mathrm{m}$, the average value of the FWHM of the intensity profile is $1.99 \pm 0.04 \mu \mathrm{m}$ (mean \pm $\mathrm{SE}$ ), coinciding with its size. For smaller bead sizes, the FWHM deviates from the actual diameter, as illustrated by the departure of the FWHM value from the equality line (Fig. 6B, dotted line). Nevertheless, $1.0-\mu \mathrm{m}$-diameter beads yield a mean FWHM of $1.21 \pm 0.02 \mu \mathrm{m}$, significantly different $(p<0.0001)$ from the $0.75 \pm 0.01 \mu \mathrm{m}$ of the $0.46 \mu \mathrm{m}$ bead. Moreover, the FWHM of the latter is significantly different $(p<0.01)$ from the FWHM of the 0.22 and $0.11 \mu \mathrm{m}$ beads $(0.65 \pm 0.02$ and $0.68 \pm 0.02 \mu \mathrm{m}$, respectively). The discrepancies between FWHM and bead diameter for beads $<1 \mu \mathrm{m}$ in diameter are not surprising given the limited resolution imposed by the finite NA objective used to project a $\sim 0.7 \mu \mathrm{m}$ diameter illumination spot (Hiraoka et al., 1990; Wilson, 1990; Castleman, 1996). Nonetheless, because the measured FWHM can be used to discriminate bead sizes of 0.5 $\mu \mathrm{m}$ and greater, we can use the calibration curve in Figure $6 B$ to estimate the actual size of the majority of the measured fluorescence domains $(0.75-3.0 \mu \mathrm{m})$.

\section{Variance analysis of single-site fluorescence transients}

Implicit in the determination of the spatial dependence of isochronal $\Delta F / F$ is the assumption that the properties of transients recorded at a single site do not vary significantly between consecutive AP stimulations. It is conceivable that a contribution to fluorescence transient variability (in addition to that described for Figs. 4, 5) could arise from AP-induced phenomena, such as the stochastic opening of $\mathrm{Ca}^{2+}$ channels or light scattering fluctuations (Obaid and Salzberg, 1996). To investigate this, we calculated the fluorescence variance as a function of time $\left({\sigma_{\mathrm{F}}}^{2}\right)$ from several AP-elicited fluorescence transients recorded while leaving the spot at the same location (see Materials and Methods). Panel $i$ in Figure $7 A$ shows 17 superimposed fluorescence transients, and their mean is shown in panel ii. Both the individual transients and their average exhibit a rapid decay, followed by a slower decay phase (DiGregorio and Vergara, 1997), typical of transients 
A
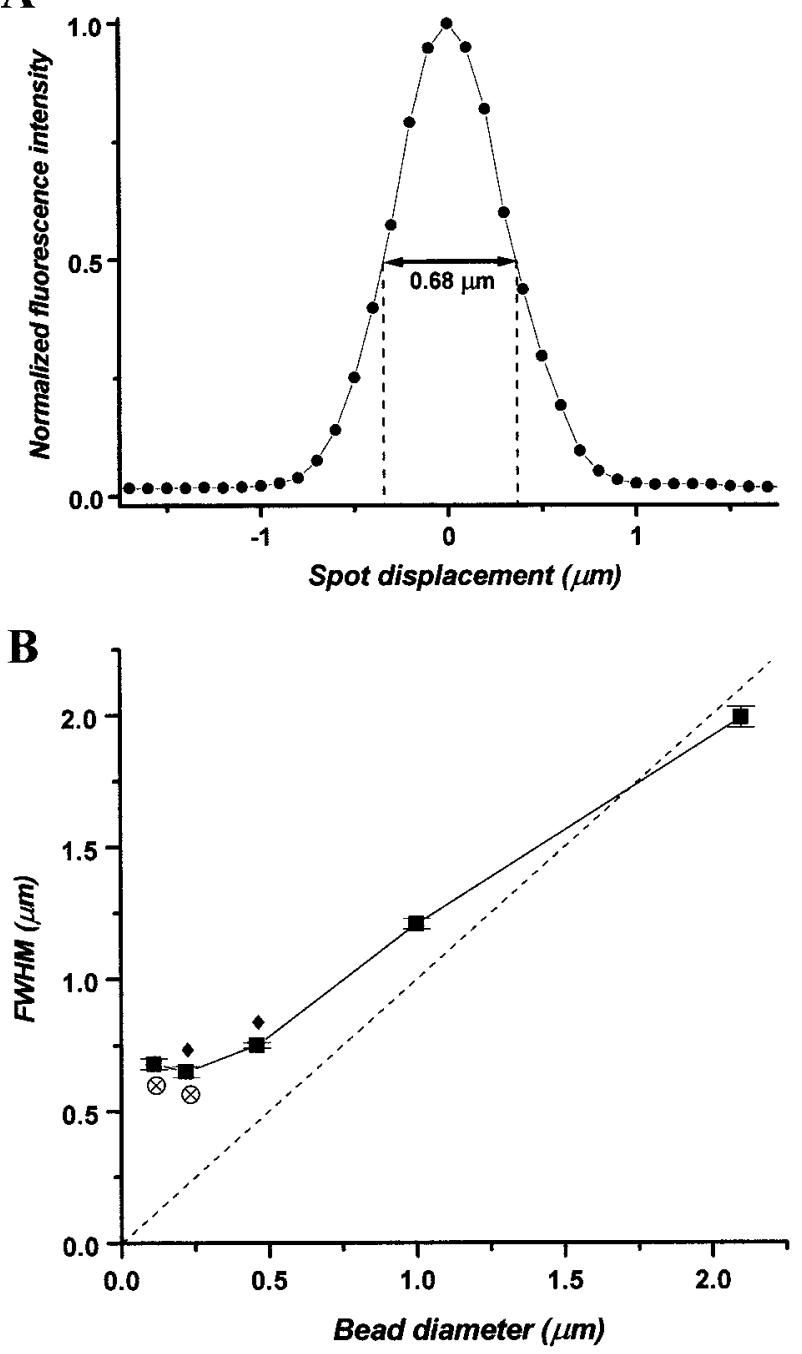

Figure 6. Calibration scans of fluorescent beads. $A$, Fluorescence intensity profile of a $0.46 \mu \mathrm{m}$ fluorescent bead. The FWHM of $0.68 \mu \mathrm{m}$ (arrow) was obtained from linear interpolation between data points. $B$, Plot of mean FWHM versus bead diameter. From small to large, the actual bead diameters were $0.11,0.22,0.46,1.0$, and $2.1 \mu \mathrm{m}$. The number of scans for each bead diameter averaged were 14, 14, 16, 9, and 11, respectively. The error bars represent the SEs in the FWHM measurements. Data points with the symbol $\otimes$ are not significantly different from each other $(p>0.2)$. Data points with the symbol $\downarrow$ are significantly different FWHM $(p<$ $0.01)$ from each other. The dotted line is drawn as a reference for $y=x$.

recorded close to the location of the maximal fluorescence change. The fluorescence variance trace in panel iii demonstrates that, after AP stimulation, there is only a negligible increase in variance above the baseline variance before the delivery of the stimuli. This suggests that AP-induced fluctuations in the fluorescence transients recorded at a single site do not further distort our estimates of the FWHM of fluorescence domains.

\section{Fluorescence gradient dissipation}

To assess the time course of dissipation of AP-induced fluorescence domains, we performed variance calculations on a set of fluorescence transients recorded at different spot locations along a scan in which a fluorescence domain was measured. Panel $i$ in Figure $7 B$ shows individual AP-elicited fluorescence transients recorded at 14 spot locations along a single scan line from a different presynaptic terminal than that in Figure $7 A$. The isochronal $\Delta F / F$ plot of that particular scan (data not shown) yielded an FWHM of $1.4 \mu \mathrm{m}$. The trace shown in panel ii of Figure $7 B$, calculated by computing the mean from fluorescence transients at each of the 14 locations, represents the mean fluorescence increase over the scanned distance (in this case, $2.8 \mu \mathrm{m}$ ). The fast decay phase clearly present in the larger transients (Fig. 7B, panel $i$ ) is not seen in the mean trace (Fig. $7 B$, panel $i i$ ) because of the averaging process with slower transients. However, the spacedependent variance trace in panel iii (Fig. 7B) displays a transient increase early after AP-stimulation, near the time when the larger transients peak. This robust increase in variance decays rapidly toward baseline within $8 \mathrm{msec}$, as shown in panel iii (Fig. 7B). The time course of the spatial variance trace represents the rapid formation and dissipation of detectable AP-induced fluorescence gradients within the presynaptic terminal. To quantitate the dissipation rate of the gradient in terms of $\Delta F / F$ (rather than its square), we fitted the square root of the variance traces (data not shown) with single exponential decay functions and obtained an average time constant for gradient dissipation of $1.4 \pm 0.6 \mathrm{msec}$ (mean $\pm \mathrm{SD} ; n=8$ ). Note that the spatially averaged trace (Fig. $7 B$, panel $i i)$ was significantly above baseline well after the dissipation of the gradients and decayed slowly toward baseline as the overall $\left[\mathrm{Ca}^{2+}\right]$ returned to its resting value.

\section{Model simulations of fluorescence domains using a diffusion-reaction model}

To better understand the relationship between the size of measured OGB-5N fluorescence domains and that of the underlying $\mathrm{Ca}^{2+}$ domain resulting from the entry of $\mathrm{Ca}^{2+}$ ions into the nerve terminal at a discrete site, we used a mathematical diffusion-reaction model (see Materials and Methods). Figure $8 \mathrm{~A}$ shows the results of a simulation designed to predict the amplitude, kinetic features, and spatial dependence of the fluorescence domain illustrated in Figures 3 and 4. The endogenous buffer concentration and the total number of channels within the entry site were set to match the amplitude and time course of the largest fluorescence transient (Fig. 3, see superposition of dashed black trace and red trace). The $x$-dimension of the $\mathrm{Ca}^{2+}$ entry site (Fig. 2) was set such that the FWHM of the isochronal $\Delta F / F$ plot of the simulated traces approximated that of the experimental isochronal $\Delta F / F$ plot.

The amplitude and time course of the $\mathrm{Ca}^{2+}$ current used to generate the simulated $\Delta F / F$ traces is shown as the black trace in panel $i$ of Figure $8 A$. Twenty-eight channels were distributed evenly over a $\mathrm{Ca}^{2+}$ entry site with an $x$-dimension of $1.1 \mu \mathrm{m}$ and a $y$-dimension of $0.5 \mu \mathrm{m}$. The number of open channels necessary to produce the measured peak $\Delta F / F$ values was in the range of the total number of $\mathrm{Ca}^{2+}$ channels estimated in active zones of bullfrog's sacculus hair cells ( 90) (Roberts et al., 1990) and crayfish synaptic boutons $(\sim 13)$ (Cooper et al., 1996). The largest (red) trace in panel $i$ of Figure $8 A$ illustrates a simulation in which the spot-detection volume was "centered" with respect to the $\mathrm{Ca}^{2+}$ entry site. The peak amplitude of this trace is 0.3 , similar to that of the largest experimental trace (Fig. 3, see superposition of dashed black trace and red trace). The simulated trace also exhibits the kinetic characteristics (rapid rise and decay, followed by a slower decay) seen in OGB-5N fluorescence traces. The simulated spot-detection volume was then displaced by $200 \mathrm{~nm}$ to attain the next largest transient (green), then another $200 \mathrm{~nm}$ to attain the blue trace, and so on. As in the experimental fluorescence traces, there is a significant spatial dependence in the peak 
$\mathbf{A}$
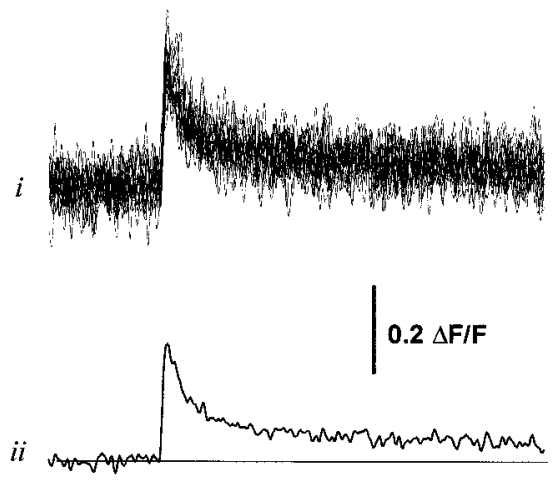

$0.006(\Delta F / F)^{2}$

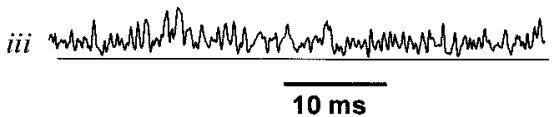

B

Scanned spot detection
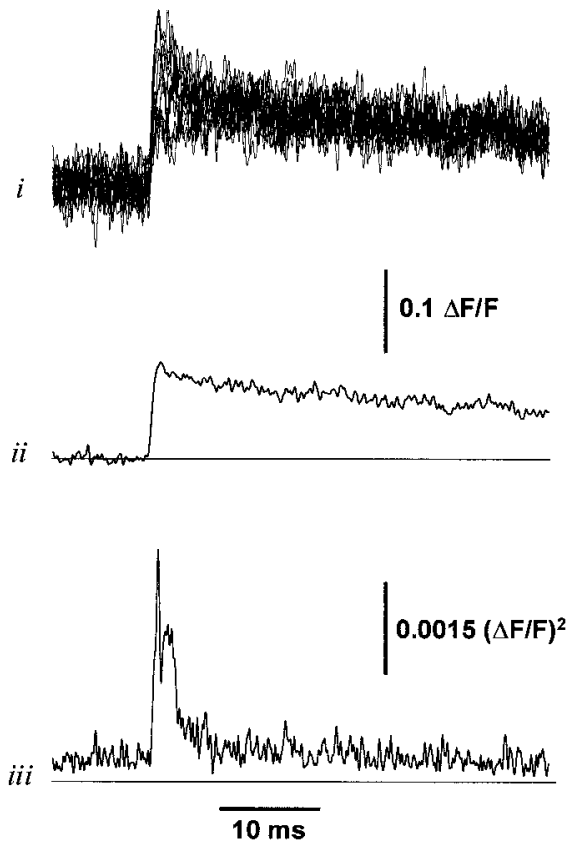

Figure 7. Fluorescence variance of stationary spot- and scan spot-detected fluorescence transients. $A$, Seventeen AP-induced fluorescence transients recorded from a single spot location (traces in $i$ ). For this experiment, 300 $\mu \mathrm{M}$ OGB-5N and $50 \mu \mathrm{M}$ EGTA were used in the pipette solution. The traces were filtered on-line at $1 \mathrm{kHz}$. The average of the stationary records (trace in ii) is plotted in the middle. The fluorescence variance (trace in iii) was calculated according to Equation 2 (see Materials and Methods). B, Fourteen AP-induced fluorescence traces (traces in $i$ ) recorded in a different nerve terminal from locations separated by $200 \mathrm{~nm}$. This terminal was loaded with $600 \mu \mathrm{M}$ OGB-5N and $50 \mu \mathrm{M}$ EGTA. Transients were filtered on-line at $1.5 \mathrm{kHz}$. Trace in ii is the mean, and trace in iii is the variance of the scanned fluorescence traces. amplitude and time course of the simulated traces: the fast decay phase was reduced, and the time-to-peak increased (Fig. 3B, compare bottom black dashed trace, purple trace).

The three-dimensional plot (Fig. 8A, panel ii) illustrates a domain comparable to that in Figure $3 C$. However, to obtain a quantitative comparison between the size of the simulated fluorescence domain and that of the experimental domain, we performed an isochronal $\Delta F / F$ analysis (Fig. $8 A$, panel iii) of the simulated traces. The resulting gaussian fit yielded an FWHM of $1.14 \mu \mathrm{m}$, approximately equal to the longest dimension of the $\mathrm{Ca}^{2+}$ entry site $(1.1 \mu \mathrm{m})$ and similar to the size of the fluorescence domain illustrated in Figures 3 and $4(1.17 \mu \mathrm{m})$.

To explore the sensitivity of the model to changes in the size of the $\mathrm{Ca}^{2+}$ entry site size, we performed a simulation identical to that in Figure $8 A$, except that we decreased the size of the $\mathrm{Ca}^{2+}$ entry site to $0.5 \times 0.5 \mu \mathrm{m}$, while preserving the density of $\mathrm{Ca}^{2+}$ channels (total number of 13). Figure $8 B$ illustrates that the resulting domain was narrower than that shown in Figure $8 A$. The individual modeled traces corresponding to the centered and $0.6-\mu \mathrm{m}$-displaced location are plotted (for comparison with the data) as top and bottom dashed traces, respectively, in Figure $5 A$. The isochronal $\Delta F / F$ plot (Fig. $8 B$, panel $i i$ ) yielded an FWHM of $0.80 \mu \mathrm{m}$, which, although smaller than the domain simulated in Figure $8 A$, is larger than the actual dimension of the small $\mathrm{Ca}^{2+}$ entry site. Nonetheless, this simulated domain approximates the size of fluorescence domain illustrated in Figure 5.

So far, we have demonstrated that the diffusion-reaction model is able to predict the spatial dependence and time course of experimentally recorded AP-induced fluorescence transients. We now further explore the relationship between the FWHM of simulated domains and the size of the $\mathrm{Ca}^{2+}$ entry site. Because simulated transients are noiseless, we were no longer constrained to using gaussian fits to estimate the FWHMs of isochronal $\Delta F / F$ plots. Consequently, we directly calculated the FWHMs from linearly interpolated plots. In Figure $8 C$ (filled circles), we plot the FWHMs of isochronal $\Delta F / F$ plots versus the actual sizes of entry sites when the $x$-dimension of the latter was varied from 0.1 to $2.1 \mu \mathrm{m}$. It can be observed that, for $\mathrm{Ca}^{2+}$ entry sites greater than $1.1 \mu \mathrm{m}$, the FWHM of the isochronal $\Delta F / F$ plot predicts accurately the dimension of the site (parallel to the direction of scan). However, as the entry site was made smaller, the FWHM deviated from the actual size and reached an asymptote of $\sim 0.7$ $\mu \mathrm{m}$, which is the size of the detection volume used in these simulations. Therefore, the FWHM of isochronal $\Delta F / F$ plots can be used as a direct measure of the $\mathrm{Ca}^{2+}$ entry site for sizes greater than $1.1 \mu \mathrm{m}$. For sites smaller than this, the deviation from linearity in Figure $8 C$ (filled circles) is reminiscent of the limitation in the optical resolution illustrated in fluorescence bead scans (Fig. 6B). Additional simulations (Fig. 8C, open circles) verified that a reduction in the $y$-dimension of the spot-detection volume to $0.1 \mu \mathrm{m}$ extended the range of linearity such that the deviation of the FWHM was reduced to $7 \%$ for a $0.7 \mu \mathrm{m} \mathrm{Ca}{ }^{2+}$ entry site and $20 \%$ for a $0.5 \mu \mathrm{m}$ entry site. Although $0.1 \mu \mathrm{m}$ is a limit of resolution not attainable experimentally, these model simulations illustrate an optimal condition in which the $x$-dimension of the detection volume is as small as the voxel size. In this case, the differences between the dimension of the predicted fluorescence domain and that of the $\mathrm{Ca}^{2+}$ entry site are not affected by the detection volume but reflect the diffusion of $\mathrm{Ca}^{2+}$ (or $\mathrm{Ca}^{2+}$-bound indicator) away from the $\mathrm{Ca}^{2+}$ entry site (Fig. 9).

We explicitly tested the model (simulations not shown) to explore the sensitivity of the FWHM to other parameters. We found that doubling the number of channels while maintaining the size of the $\mathrm{Ca}^{2+}$ entry site, a twofold increase in $D_{2}$ (OGB$5 \mathrm{~N}$ ), a threefold increase in $D_{\mathrm{Ca}}$, and a 100 -fold reduction in the concentration of OGB-5N did not affect the domain FWHM. However, the time course and amplitude of the predicted fluorescence transients departed from that of the experimental data.

We used model simulations to predict nonmeasurable quantities, such as the magnitude of fluorescence and $\left[\mathrm{Ca}^{2+}\right]$ changes in domains close to the membrane. In Figure $9 A$ (panel $i$ ), we plot 


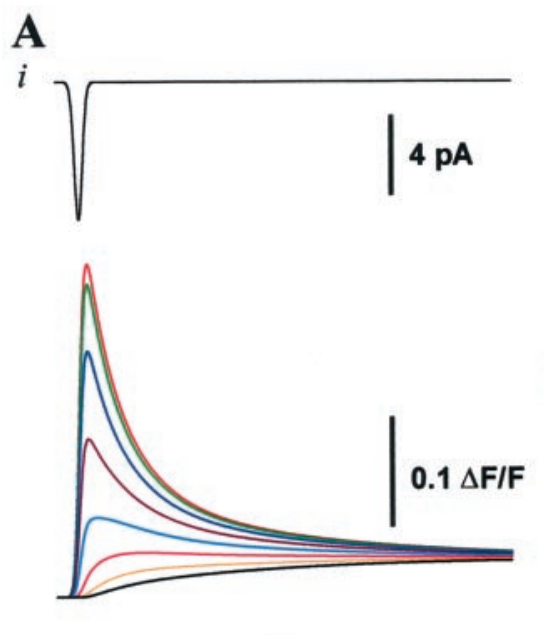

$i i$

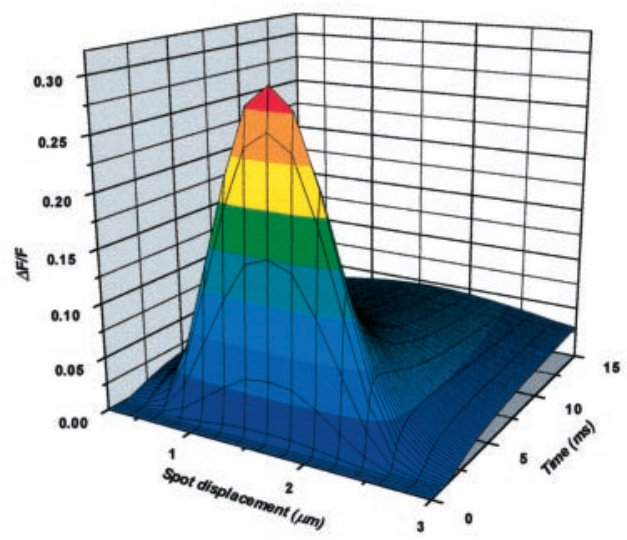

$5 \mathrm{~ms}$

B

$i$

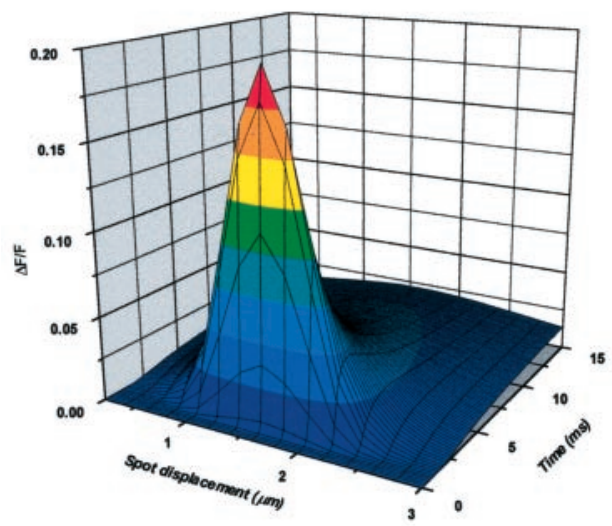

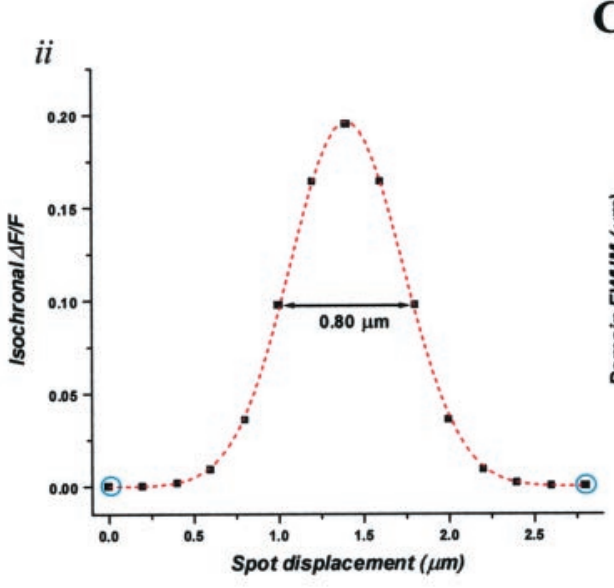

iii

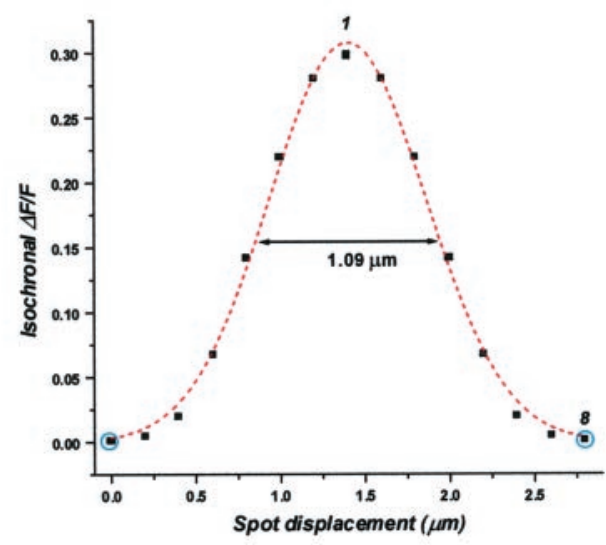

C

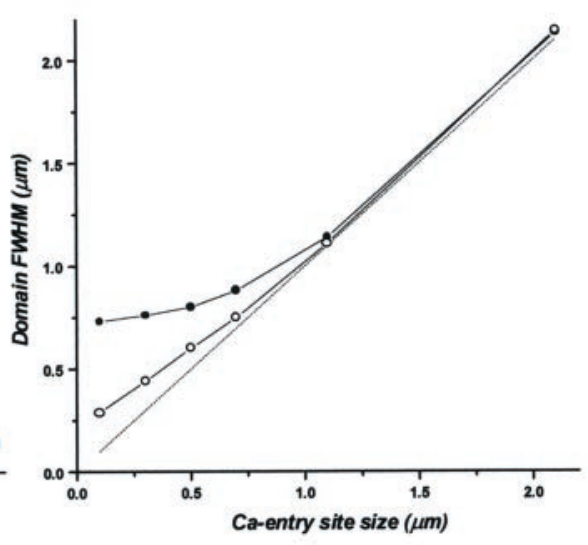

Figure 8. Model simulations of scanned fluorescence data. A, Results from a diffusion-reaction simulation in which the 28 channels were evenly distributed in a "checkerboard" pattern spanning an area of 1.1 ( $x$-dimension) $\times 0.5$ ( $y$-dimension) $\mu \mathrm{m}$ with an elementary cell of $0.1 \times 0.1 \mu \mathrm{m}$. The top trace in $i$ represents the simulated $\mathrm{Ca}^{2+}$ current. The bottom traces are the simulated $\Delta F / F$ transients. The OGB-5N concentration was set to $600 \mu \mathrm{M}$, and the EGTA concentration was set to $50 \mu \mathrm{M}$. $i i$, Simulated fluorescence traces are plotted according to the relative location of the detection volume. iii, Isochronal $\Delta F / F$ plot obtained following the same procedure as for the experimental data. The plot was fit to a gaussian function (red dashed curve) using the data points bracketed by those identified by aqua circles (FWHM, $1.09 \mu \mathrm{m}$; arrow). The points labeled 1 and 8 correspond to isochronal data obtained from red to black trace in $A$. $B$, Simulation identical to that in $A$, except the area of the calcium entry site was reduced to $0.5 \times 0.5 \mu \mathrm{m}$ while preserving the density of channels (13 channels). All other model parameters are identical to those simulations in $A$. $i$, Individual fluorescence traces plotted according to the simulated spot location. $i i$, Isochronal $\Delta F / F$ plot fitted with a gaussian (red dashed curve) of $0.80 \mu \mathrm{m} F \mathrm{FHM}$ (arrow). $C$, Plot of simulated FWHMs versus the size of the $\mathrm{Ca}^{2+}$ entry site. The $x$ - and $y$-dimensions of the spot-detection volume were set to $0.7 \mu \mathrm{m}$, the $y$-dimension of the $\mathrm{Ca}^{2+}$ entry site was set to $0.5 \mu \mathrm{m}$, and the $x$-dimension of the $\mathrm{Ca}^{2+}$ entry site was set to $0.1,0.3,0.5,0.7,1.1$, or $2.1 \mu \mathrm{m}$. FWHMs (closed circles), obtained directly from linearly interpolated isochronal $\Delta F / F$ plots, are $0.73,0.76,0.80,0.88,1.14$, and $2.13 \mu$ m, respectively. The open circles represent FWHMs obtained from simulations using the same sizes of $\mathrm{Ca}^{2+}$ entry sites, except that the $x$-dimension of the detection volume was set to $0.1 \mu \mathrm{m}$. From small to large entry sites, the values were $0.29,0.44,0.60,0.75,1.11$, and $2.14 \mu \mathrm{m}$, respectively. The dashed line is drawn as a reference for $y=x$.

a snapshot of the spatial distribution of the AP-induced fluorescence increase within $50 \mathrm{~nm}$ of the membrane, obtained at the time of the peak of the largest fluorescence transient (Fig. 8A, panel $i$, top trace). It can be observed that the maximal $\Delta F / F$ plotted in Figure $9 A$ is considerably larger (sevenfold) than the peak of the largest scanned trace (Fig. $8 A$, panel $i$, red trace). This difference arises from the calculation of the spatial average over the detection volume implicit to simulations of scanned data in which there is a significant proportion of volume elements not contributing to the fluorescence change. The line profile centered on the $\mathrm{Ca}^{2+}$ entry site (Fig. $9 A$, panel $\mathrm{ii}$ ) illustrates that majority of the fluorescence $(\sim 90 \%)$ arises from within the boundaries of the $\mathrm{Ca}^{2+}$ entry site. The spatial profile of the $\left[\mathrm{Ca}^{2+}\right]$ (Fig. $9 B$, panel $i$ ) shows that, for the same simulation, the free $\left[\mathrm{Ca}^{2+}\right]$ attains local maxima of $\sim 6.5 \mu \mathrm{M}$ at volume elements $(0.1 \times 0.1 \times$ $0.05 \mu \mathrm{m}$ ) containing a $\mathrm{Ca}^{2+}$ channel. The line profile (Fig. $9 B$, panel $i$ ) highlights the steep $\left[\mathrm{Ca}^{2+}\right]$ gradients at the boundaries of the entry site, demonstrating that, at early times after APstimulation, the width of the $\mathrm{Ca}^{2+}$ domain is slightly narrower than the fluorescence domain and slightly broader than the extent of the entry site. The spatial profile of the $\left[\mathrm{Ca}^{2+}\right]$ within $5 \mathrm{~nm}$ of the membrane (Fig. 9B, inset), obtained using a $10 \mathrm{~nm}$ grid spacing in the computation and at the time when the $\mathrm{Ca}^{2+}$ influx peaked $(1 \mathrm{msec})$, demonstrates that the peak $\left[\mathrm{Ca}^{2+}\right]$ reached levels of $\sim 300 \mu \mathrm{M}$ in the $10 \times 10 \times 5 \mathrm{~nm}$ truncated voxel containing a $\mathrm{Ca}^{2+}$ channel. This value is within twofold of that 

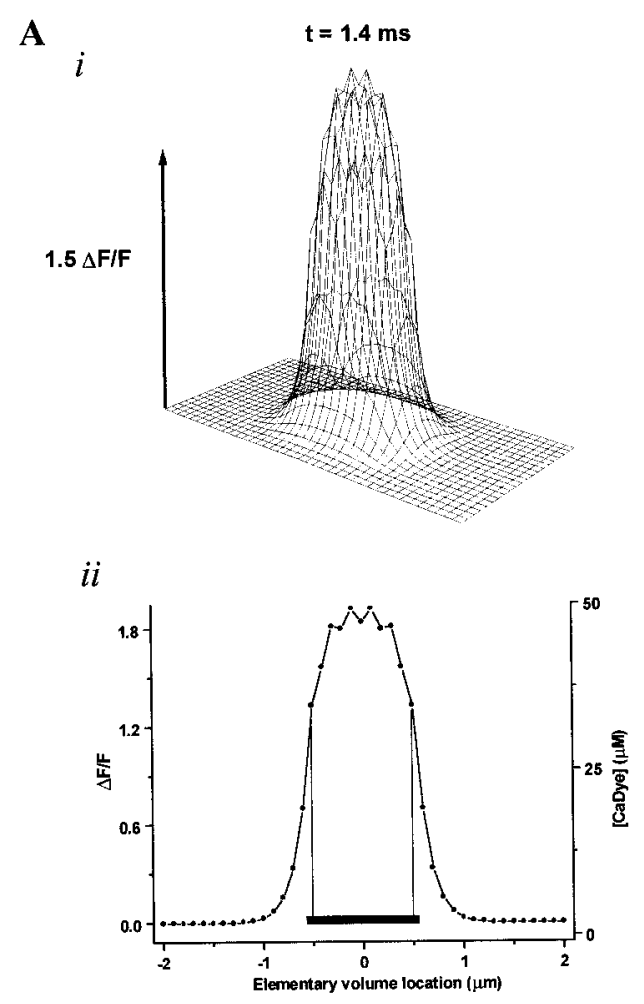
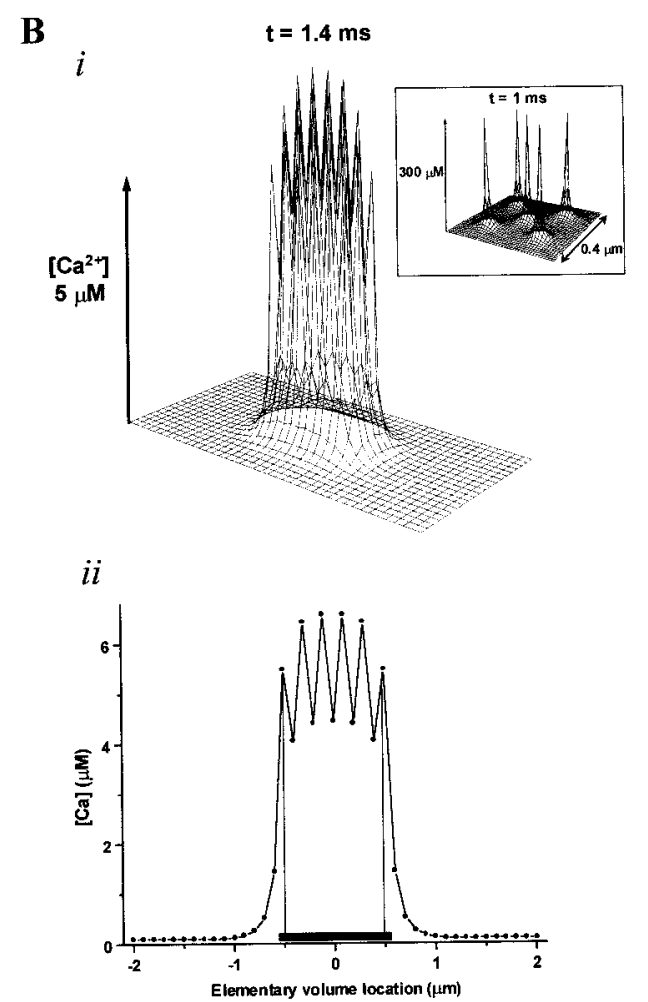

Figure 9. $\quad \Delta F / F$ and $\left[\mathrm{Ca}^{2+}\right]$ amplitude profiles adjacent to the membrane plane during the simulation in Figure $8 A$. $A, i$, Spatial profile of $\Delta F / F$ within $50 \mathrm{~nm}$ of the $x-y$ membrane plane of the model nerve terminal obtained at the isochronal point $(1.4 \mathrm{msec}$ from the start point). $i i$, Line profile of $\Delta F / F$ values along the $x$-direction bisecting the $\mathrm{Ca}^{2+}$ entry site. The thick bar indicates the $x$-dimension of the $\mathrm{Ca}^{2+}$ entry site. $B, i$, Spatial profile of $\left[\mathrm{Ca}^{2+}\right]$ within $50 \mathrm{~nm}$ of the $x-y$ membrane plane of the model nerve terminal obtained at the isochronal point. Inset, $\left[\mathrm{Ca}^{2+}\right]$ profile within $5 \mathrm{~nm}$ of the membrane obtained at a time when $\mathrm{Ca}^{2+}$ current peaked $(1 \mathrm{msec})$. The channel density and distribution pattern were unchanged. $i i$, Line profile of $\left[\mathrm{Ca}^{2+}\right]$ values along the $x$-direction bisecting the $\mathrm{Ca}^{2+}$ entry site. The thick bar indicates the $x$-dimension of the $\mathrm{Ca}^{2+}$ entry site. predicted by similar model simulations by other authors (Simon and Llinas, 1985; Yamada and Zucker, 1992; Roberts, 1994; Bertram et al., 1999).

\section{Effects of EGTA on kinetic properties and spatial dependence of fluorescence transients}

The $\mathrm{Ca}^{2+}$ chelator EGTA has been extensively used to investigate the $\mathrm{Ca}^{2+}$ dependence of fast neurotransmitter release (Adler et al., 1991; Atluri and Regehr, 1996; Borst and Sakmann, 1996; Ohana and Sakmann, 1998). In particular, its slow association rate constant (Smith et al., 1984) has been exploited to infer the spatiotemporal dependence of $\mathrm{Ca}^{2+}$-dependent phenomena (Neher, 1998). Here, we study the effects of various concentrations of EGTA on the time course and spatial dependence of AP-induced $\mathrm{Ca}^{2+}$-dependent fluorescence transients to experimentally determine whether the FWHM of measured fluorescence domains is affected by an exogenous $\mathrm{Ca}^{2+}$ buffer or whether it is mostly determined by the size of the $\mathrm{Ca}^{2+}$ entry site.

Similar to transients presented in the previous figures, an AP-induced fluorescence transient recorded in the presence of 10 $\mu \mathrm{M}$ EGTA exhibited rapid and slow decay phases (Fig. 10A). As the EGTA concentration was increased to $500 \mu \mathrm{M}$ and $2 \mathrm{~mm}$, the slow decay of the fluorescence transient was accelerated significantly (Fig. 10A). To quantify these changes in kinetics, we fit the transients using a tri-exponential fitting routine (see Materials and Methods). Table 1 summarizes the fitted results of several OGB-5N fluorescence transients recorded in the presence of various [EGTA]. It should be noted that (1) occasionally, the tri-exponential fitting routine yielded only two independent time constants rather than three, and (2) for quantitative comparisons, we pooled data from experiments in which either 10 or $50 \mu \mathrm{M}$ EGTA was added to the pipette solution and denoted this group as having low EGTA. We can best demonstrate the effects of EGTA on the kinetic properties of the transients by evaluating the number of records exhibiting a slow time constant $\left(\tau_{3}\right.$ of $>30$ msec). Twelve of fourteen averaged single site transients, recorded in the presence of low [EGTA], exhibited a significant $\tau_{3}$ (fit amplitude, $A_{3}$, greater than $10 \%$ of the peak amplitude of the transients). In the presence of $500 \mu \mathrm{M}$ EGTA, only three of eight averaged transients exhibited a significant $\tau_{3}$. In the presence of 2 mM EGTA, only one transient of 16 exhibited a significant $\tau_{3}$. The averaged second time constant $\left(\tau_{2}\right)$ decreased with increasing EGTA concentrations; however, the differences were not statistically significant $(p>0.1)$. The fastest time constant $\left(\tau_{1}\right)$ was not significantly affected by increased EGTA concentrations. Nonetheless, the relative amplitude $\left(A_{1}\right)$ of $\tau_{1}$ increased significantly when increasing the EGTA concentration, consistent with a transformation of a slower decay process into an overall faster one. Together, these data demonstrate that EGTA is most effective at reducing the contribution of slow components in the fluorescence transients, which is consistent with an inability to act as a significant $\mathrm{Ca}^{2+}$ sink until late in time because of its slow association rate constant.

To determine whether the acceleration of the rate of decay of transients induced by EGTA was associated with a significant reduction in the size of the $\mathrm{Ca}^{2+}$ domain, as assessed by the FWHM of isochronal $\Delta F / F$ plots, we performed scan experiments in the presence of various [EGTA]. The isochronal $\Delta F / F$ plot presented in Figure $10 B$ exhibits a relatively broad FWHM of $1.80 \mu \mathrm{M}$, even in the presence of $2 \mathrm{~mm}$ EGTA. Table 2 summarizes the mean FWHM as calculated from experiments performed in the presence of various [EGTA]. Neither $500 \mu \mathrm{M}$ EGTA nor 2 mM EGTA (FWHM, 1.7 and $1.5 \mu \mathrm{m}$, respectively) were able to significantly $(p>0.5)$ alter the mean FWHM (1.5 $\mu \mathrm{m})$ obtained from experiments performed in low concentrations of EGTA. Table 2 also summarizes the mean peak $\Delta F / F$ of AP-elicited OGB-5N transients recorded in the presence of various [EGTA]. There is no statistically significant difference $(p>$ 
A

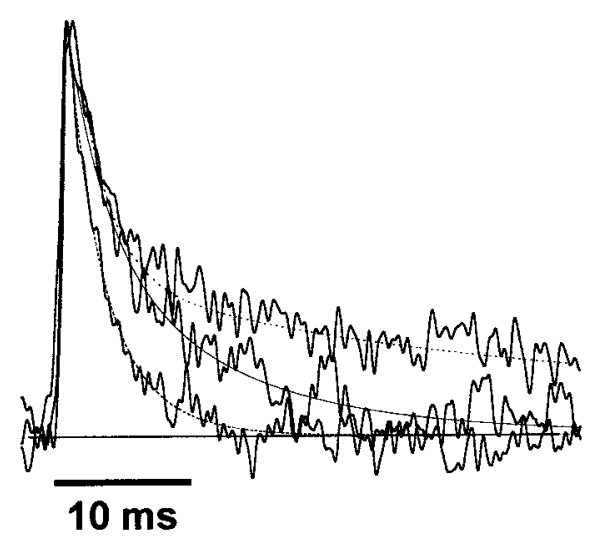

C

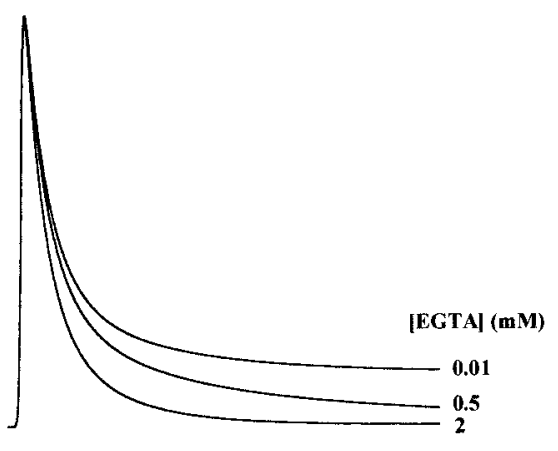

$10 \mathrm{~ms}$
B

2 mM EGTA

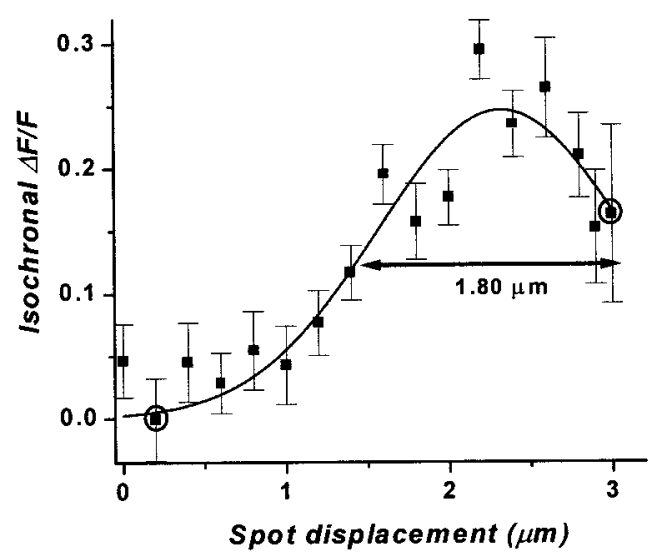

D

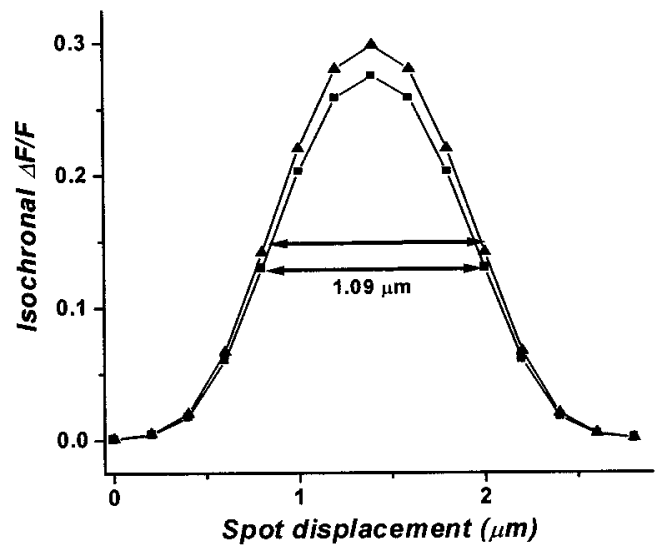

Figure 10. Acceleration in the decay of presynaptic AP-induced OGB-5N fluorescence transients by EGTA without an effect on the measured domain size. $A$, Normalized plot of single-site fluorescence transients recorded in the presence of various concentrations of EGTA in the pipette solution. The short dashed trace is a bi-exponential function, with the fitted parameters $\tau_{1}$ of $2.7 \mathrm{msec}$ and $\tau_{2}$ of $33.9 \mathrm{msec}$, and $A_{1}$ of 0.69 and $\mathrm{A}_{2}$ of 0.31 used to identify its corresponding transient recorded in the presence of $10 \mu \mathrm{M}$ EGTA (average of 5). The thin solid trace is a tri-exponential function ( $\tau_{1}$ of 2.6 msec; $\tau_{2}$ of $7.5 \mathrm{msec} ; \tau_{3}$ of $13 \mathrm{msec} ; \mathrm{A}_{1}$ of $0.45 ; \mathrm{A}_{2}$ of 0.27 ; and $\mathrm{A}_{3}$ of 0.27 ) corresponding to a transient recorded from a different terminal in the presence of 500 $\mu \mathrm{M}$ EGTA (average of 4). The long dashed trace is a bi-exponential ( $\tau_{1}$ of $1.7 \mathrm{msec} ; \tau_{2}$ of $3.5 \mathrm{msec} ; \mathrm{A}_{1}$ of 0.44 ; and $\mathrm{A}_{2}$ of 0.56$)$ identifying the averaged $(\mathrm{n}=5)$ fluorescence transient recorded from a third nerve terminal in the presence of $2 \mathrm{mM}$ EGTA. The baseline $(0 \Delta F / F)$ is illustrated by the dotted line. $B$, The isochronal $\Delta F / F$ plot of a family of scanned fluorescence transients recorded in the presence of 2 mM EGTA. The plot was fit with a gaussian (between the circled data points) yielding an FWHM (arrow) of $1.80 \mu \mathrm{m}$. Data points represent a time average within $\pm 0.39 \mathrm{msec}$ of the isochronal point. $C$, Model simulations of fluorescence transients obtained when the detection volume was centered on the $\mathrm{Ca}^{2+}$ entry site. Simulations were identical to those in Figure $8 A$, except that the EGTA concentrations were set to match the experimental conditions in Figure $10 A$. The transients were normalized to their peak values. $D$, Simulated isochronal $\Delta F / F$ plots obtained using $10 \mu \mathrm{M}$ EGTA (triangles) and 2 mM EGTA (squares). Note that the FWHMs (arrows) are both $1.09 \mu \mathrm{m}$.

0.2 ) among all the peak $\Delta F / F$ values obtained at low, medium, and high concentrations of EGTA.

\section{Model simulations in the presence of various [EGTA]}

To provide theoretical evidence that EGTA can accelerate the decay of AP-induced fluorescence transients without altering their peak amplitude or the FWHM of isochronal $\Delta F / F$ plots, we performed simulations identical to those in Figure $8 A$, except that the EGTA concentration was varied. Figure $10 C$ shows simulated centered fluorescence transients when the [EGTA] was set to 10 $\mu \mathrm{M}, 500 \mu \mathrm{M}$, and $2 \mathrm{mM}$. In the presence of $10 \mu \mathrm{M}$ EGTA, the slow decay component is prominent, whereas at higher [EGTA] the acceleration observed in experimental traces (Fig. 10A) is well reproduced. Moreover, consistent with the experimental data, there is no detectable difference in the FWHM of isochronal $\Delta F / F$ plots when comparing the results from a simulation performed in the presence of $10 \mu \mathrm{M}$ EGTA with those in $2 \mathrm{~mm}$ EGTA (Fig. 10D). The model does predict an $8 \%$ reduction in the peak $\Delta F / F$ that we cannot detect experimentally given the noise magnitude.

\section{DISCUSSION}

Here, we demonstrate that the confocal spot-detection method (Escobar et al., 1994), when used in combination with a lowaffinity $\mathrm{Ca}^{2+}$ indicator (DiGregorio and Vergara, 1997) and a high-resolution stage-scan device, can be used to characterize the rapid formation and dissipation of fluorescence domains in re- 


\begin{tabular}{|c|c|c|c|c|c|c|}
\hline [EGTA] & $\tau_{1}$ & $A_{1}$ & $\tau_{2}$ & $A_{2}$ & $\tau_{3}$ & $A_{3}$ \\
\hline \multicolumn{7}{|l|}{$10 / 50 \mu \mathrm{M}$} \\
\hline $\begin{array}{l}\text { mean } \pm \mathrm{SD} \\
n / N\end{array}$ & $\begin{array}{l}* 1.7 \pm 1.0 \\
14 / 14\end{array}$ & $* * 0.49 \pm 0.2$ & $\begin{array}{l}{ }^{\#} 14 \pm 11 \\
8 / 14\end{array}$ & $0.44 \pm 0.2$ & $\begin{array}{l}74 \pm 54 \\
12 / 14\end{array}$ & $0.30 \pm 0.1$ \\
\hline \multicolumn{7}{|l|}{$500 \mu \mathrm{M}$} \\
\hline $\begin{array}{l}\text { mean } \pm \mathrm{SD} \\
n / N\end{array}$ & $\begin{array}{l}* 1.6 \pm 1.0 \\
8 / 8\end{array}$ & $0.49 \pm 0.2$ & $\begin{array}{l}{ }_{8 / 8}^{*} 11 \\
8 / 8\end{array}$ & $0.44 \pm 0.2$ & $\begin{array}{l}35 \pm 26 \\
3 / 8\end{array}$ & $0.19 \pm 0.1$ \\
\hline \multicolumn{7}{|l|}{$2 \mathrm{~mm}$} \\
\hline $\begin{array}{l}\text { mean } \pm \mathrm{SD} \\
n / N\end{array}$ & $\begin{array}{l}* 1.8 \pm 0.5 \\
15 / 16\end{array}$ & $* * 0.69 \pm 0.2$ & $\begin{array}{l}{ }^{\# 9} \pm 7 \\
13 / 16\end{array}$ & $0.41 \pm 0.2$ & $\begin{array}{c}44.5 \\
1 / 16\end{array}$ & 0.33 \\
\hline
\end{tabular}

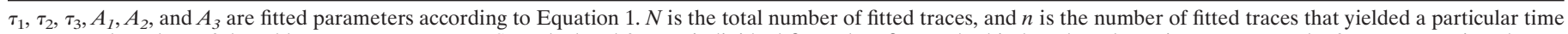

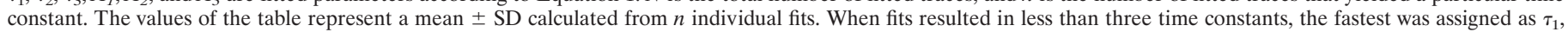

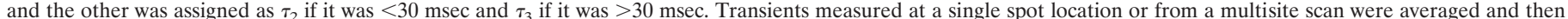

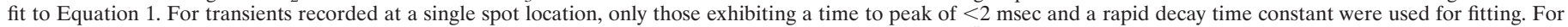
scanned multisite recordings, the largest three or four transients of a scan were averaged before fitting. ${ }^{* \#} p>0.5 ;{ }^{* *} p<0.2 ;$ Student's $t$ test.

\begin{tabular}{|c|c|c|c|}
\hline [EGTA] & $10 / 50 \mu \mathrm{M}$ & $500 \mu \mathrm{M}$ & $2 \mathrm{~mm}$ \\
\hline \multicolumn{4}{|l|}{ FWHM $(\mu \mathrm{m})$} \\
\hline mean $\pm \mathrm{SD}$ & $* 1.54 \pm 0.69$ & $* 1.69 \pm 0.58$ & $* 1.51 \pm 0.52$ \\
\hline$n$ & 9 & 3 & 9 \\
\hline \multicolumn{4}{|l|}{ Peak $\Delta F / F$} \\
\hline mean $\pm \mathrm{SD}$ & $* * 0.14 \pm 0.06$ & $* * 0.18 \pm 0.13$ & $* * 0.12 \pm 0.05$ \\
\hline$n$ & 16 & 7 & 17 \\
\hline
\end{tabular}

Peak $\Delta F / F$ is the averaged maximum $\Delta F / F$ of transients measured at a single spot location or the averaged maximum $\Delta F / F$ of the three or four largest transients from a scan. Only traces exhibiting a time-to-peak of $<2 \mathrm{msec}$ and a rapid decay time constant were used for further analysis of peak $\Delta F / F$. ${ }^{*} p>0.7$; ** $p>0.2$; Student's $t$ test.

sponse to single APs. We find that OGB-5N fluorescence transients recorded in a single scan, that is from a series of spot locations separated by 200-300 nm, exhibit dramatic changes in their magnitude and time courses. Three dimensional plots of a family of scanned fluorescence transients against the spot displacement show the existence of AP-induced fluorescence domains. We demonstrate that the measurement of the FWHM of an isochronal $\Delta F / F$ plot, at early times after AP invasion, provides an estimate of the $\mathrm{Ca}^{2+}$ domain size. Moreover, mathematical model simulations suggest that the FWHM of the isochronal $\Delta F / F$ plots can be used to estimate the size of the $\mathrm{Ca}^{2+}$ entry site. Consistent with this prediction, domains measured in the presence of high EGTA did not exhibit altered FWHM.

In agreement with a previous report (DiGregorio and Vergara, 1997), the time course of the largest OGB-5N fluorescence transients of individual scans exhibited a typical rapid rising phase, followed by a fast and slow decay toward baseline. Using low EGTA concentrations in the pipette solution, we found here that the decay of these fluorescence transients were best fitted with three time constants: a fast ( $\tau_{1}$ of $\left.1.7 \mathrm{msec}\right)$, an intermediate $\left(\tau_{2}\right.$ of $16 \mathrm{msec}$ ), and a slow $\left(\tau_{3}\right.$ of $78 \mathrm{msec}$ ) time constant (Fig. 10A). Model simulations (Fig. $8 A$ ) illustrate that the largest transients occur at locations at which the detection spot was centered on a site of $\mathrm{Ca}^{2+}$ entry and that their time course most closely mimics this multicomponent decay. Although the use of a tri-exponential fitting routine seems to be sufficient to describe the overall decay of these fluorescence transients, we do not expect each time constant to represent a specific underlying physical process. Tran- sients recorded at progressively more distant, or off-center locations, exhibited slower rates of rise, marked reductions in their peak values, and a loss of the rapid decay component (Fig. 4A, , traces $c-f)$. These spatially dependent characteristics were indicative of the diff usion-dependent dissipation of fluorescence gradients, which we found to collapse on average within $\sim 7 \mathrm{msec}$. This dissipation time is similar to that observed previously for intrasarcomeric $\mathrm{Ca}^{2+}$ indicator fluorescence transients in skeletal muscle fibers (Escobar et al., 1994; Monck et al., 1994). However, it is slower than the $\sim 800 \mu \mathrm{sec}$ duration of an aequorin emission domain observed in a squid presynaptic terminal (Sugimori et al., 1994). The dissipation time is faster than the $\sim 15 \mathrm{msec}$ measured using OGB-5N in lizard presynaptic boutons stimulated with 3,4 diaminopyridine (DAP)-broadened APs (David et al., 1997). In this latter case, the discrepancy may be a result of intrinsic differences between preparations (e.g., size of the presynaptic terminals and properties of endogenous $\mathrm{Ca}^{2+}$ buffers) or of the prolongation of the $\mathrm{Ca}^{2+}$ entry by DAP.

The slow decay phase, common to all scanned OGB-5N fluorescence transients in the presence of low [EGTA], is similar to that of AP-induced $\mathrm{Ca}^{2+}$ transients recorded using low-affinity indicators in whole terminal or multisynaptic preparations (Regehr and Atluri, 1995; Feller et al., 1996; Helmchen et al., 1997; Sinha et al., 1997). Because this slow decay phase occurs after the collapse of the $\mathrm{Ca}^{2+}$ gradients, as assessed from the variance and mean traces (Fig. $7 B$, panels ii, iii), it could represent $\mathrm{Ca}^{2+}$ binding to slow $\mathrm{Ca}^{2+}$ buffers, a slow $\mathrm{Ca}$ extrusion-uptake mechanism, or both. Our results demonstrate that, as expected from theoretical predictions (Fig. 10C) (Markram et al., 1998), $500 \mu \mathrm{M}$ and $2 \mathrm{~mm}$ [EGTA] yield a significant acceleration in the long decay time constant $\left(\tau_{3}\right)$ of the transients (Fig. 10C, Table 1). Interestingly, EGTA produces a similar acceleration in the decay rate of spatially averaged fluorescence transients detected in other presynaptic preparations (Atluri and Regehr, 1996; Feller et al., 1996; Markram et al., 1998).

Given the limitations of our detection method, how can the FWHM of measured fluorescence domains be used to estimate the size of the $\mathrm{Ca}^{2+}$ entry site? We address this question by referring to the fluorescent bead calibrations and model simulations. When pooling together measurements of FWHM from experiments performed in low and high [EGTA] (Table 2), they ranged from 0.62 to $3.0 \mu \mathrm{m}$, with a mean value of $1.6 \pm 0.6 \mu \mathrm{m}$ $(n=21)$. According to the calibration curve (Fig. $7 B)$, the smallest domain FWHM would correspond to an undefined 
diameter of $<0.5 \mu \mathrm{m}$. The other 20 measured domains (FWHM, $0.75-3.0 \mu \mathrm{m}$ ) could be adjusted according to the calibration curve such that the "true" dimensions of the fluorescence domains ranged from 0.5 to $3 \mu \mathrm{m}$. Model simulations predict (Fig. $8 C$, open circles) that this range of fluorescence domain dimensions correspond, with better than $25 \%$ accuracy, to the size of the $\mathrm{Ca}^{2+}$ entry site. The accuracy improves dramatically for larger domains such that, at $1.0 \mu \mathrm{m}$, the error is reduced to $<2 \%$. However, this improved accuracy does not extend indefinitely because of the limitation of using gaussian fits of experimental data to estimate their FWHM. For example, model simulations show that, for a $2.1 \mu \mathrm{m} \mathrm{Ca}{ }^{2+}$ entry site, there is a $7 \%$ underestimation of the FWHM when using a Guassian fit compared with the FWHM of the linearly interpolated plot. This difference is primarily caused by the inadequacy of the gaussian function in representing the geometrical properties of isochronal $\Delta F / F$ plots from large domains.

Interestingly, this range of $\mathrm{Ca}^{2+}$ entry site sizes is consistent with the 1-3 $\mu \mathrm{m}$ range in diameter of fluorescence spots observed in this preparation, using the synaptic vesicle membrane dye FM1-43 (Dai and Peng, 1995). In addition, electron microscopy studies have shown that active zones $(0.2-0.5 \mu \mathrm{m}$ in diameter) tend to cluster within areas of neuronal contact spanning greater than $1 \mu \mathrm{m}$ (Weldon and Cohen, 1979; Buchanan et al., 1989; Dai and Peng, 1995). Thus, it is likely that the $\mathrm{Ca}^{2+}$ entry sites studied here represent these multiple neighboring active zones.

What is the basis for the inability of EGTA (up to $2 \mathrm{~mm}$ ) to reduce the FWHM of fluorescence domains, as demonstrated by both experimental data and model simulations? It has been proposed (Pape et al., 1995; Naraghi and Neher, 1997) that, at steady state, a length constant $\left.\left[\lambda=\sqrt{D_{C a} /\left(k_{E G T A}^{+} \cdot[E G T A]\right.}\right)\right]$ can describe the range of action of EGTA on $\mathrm{Ca}^{2+}$ ions with respect to an entry site in which $k_{\mathrm{EGTA}}^{+}$is the EGTA association rate constant. Using our model parameters, we calculate $\lambda$ to be $0.13 \mu \mathrm{m}$. Thus, at steady state and for a point source, $2 \mathrm{~mm}$ EGTA would be expected to reduce the $\mathrm{Ca}^{2+}$ domain to $\sim 37 \%$ of its maximum value at a radial distance $0.13 \mu \mathrm{m}$ of the source. Because $2 \mathrm{~mm}$ EGTA did not reduce the size of measured fluorescence domains, the above calculation implies that, in the absence of EGTA, $\mathrm{Ca}^{2+}$ diffusion was already constrained to $<0.13 \mu \mathrm{m}$ from the boundaries of an extended entry site. This is likely caused by the presence of a fixed buffer, as suggested by model simulations (data not shown).

We have demonstrated that, in Xenopus cultured nerve terminals, $\mathrm{Ca}^{2+}$ channels are clustered within discrete regions measuring up to $3 \mu \mathrm{m}$ in one dimension. Although the pattern of channel distribution within these regions is not known, theoretical predictions suggest that the proximity of channels to each other at release sites impact the $\left[\mathrm{Ca}^{2+}\right]$ profile mediating synaptic transmission (Neher, 1998; Bertram et al., 1999). In our model simulations, we distributed the channels evenly as a first approximation. Other patterns of channel distribution may also predict our data provided that (1) the overall dimension of the $\mathrm{Ca}^{2+}$ entry site matches that of the adjusted FWHM fluorescence domains, and (2) their separation distance between channels is not so large that the isochronal $\Delta F / F$ profile deviates from a single gaussian-like shape. It is likely that an improved $\mathrm{S} / \mathrm{N}$ ratio in the fluorescence transients and a reduction of the detection volume may allow us to resolve structures as small as individual active zones.

\section{REFERENCES}

Adler EM, Augustine GJ, Duffy SN, Charlton MP (1991) Alien intracellular calcium chelators attenuate neurotransmitter release at the squid giant synapse. J Neurosci 11:1496-1507.

Allbritton NL, Meyer T, Stryer L (1992) Range of messenger action of calcium ion and inositol 1,4,5-trisphosphate. Science 258:1812-1815.

Atluri PP, Regehr WG (1996) Determinants of the time course of facilitation at the granule cell to Purkinje cell synapse. J Neurosci 16:5661-5671.

Augustine GJ, Charlton MP, Smith SJ (1987) Calcium action in synaptic transmitter release. Annu Rev Neurosci 10:633-693.

Barrett EF, Stevens CF (1972) The kinetics of transmitter release at the frog neuromuscular junction. J Physiol (Lond) 227:691-708.

Bertram R, Smith GD, Sherman A (1999) Modeling study of the effects of overlapping $\mathrm{Ca}^{2+}$ microdomains on neurotransmitter release. Biophys J 76:735-750.

Borst JG, Sakmann B (1996) Calcium influx and transmitter release in a fast CNS synapse. Nature 383:431-434.

Buchanan J, Sun YA, Poo MM (1989) Studies of nerve-muscle interactions in Xenopus cell culture: fine structure of early functional contacts. J Neurosci 9:1540-1554.

Castleman KR (1996) Digital image processing. Englewood Cliffs, NJ: Prentice Hall.

Chad JE, Eckert R (1984) Calcium domains associated with individual channels can account for anomalous voltage relations of $\mathrm{Ca}$-dependent responses. Biophys J 45:993-999.

Church PJ, Stanley EF (1996) Single L-type calcium channel conductance with physiological levels of calcium in chick ciliary ganglion neurons. J Physiol (Lond) 496:59-68.

Cohen MW, Rodriguez-Marin E, Wilson EM (1987) Distribution of synaptic specializations along isolated motor units formed in Xenopus nerve-muscle cultures. J Neurosci 7:2849-2861.

Cooper RL, Winslow JL, Govind CK, Atwood HL (1996) Synaptic structural complexity as a factor enhancing probability of calciummediated transmitter release. J Neurophysiol 75:2451-2466.

Dai Z, Peng HB (1995) Presynaptic differentiation induced in cultured neurons by local application of basic fibroblast growth factor. J Neurosci 15:5466-5475.

David G, Barrett JN, Barrett EF (1997) Stimulation-induced changes in $\left[\mathrm{Ca}^{2+}\right]$ in lizard motor nerve terminals. J Physiol (Lond) 504:83-96.

DiGregorio DA, Vergara JL (1997) Localized detection of action potential-induced presynaptic calcium transients at a Xenopus neuromuscular junction. J Physiol (Lond) 505:585-592.

DiGregorio DA, Marengo J, Peskoff A, Vergara JL (1998) Measurement of presynaptic calcium microdomains in a cultured neuromuscular junction. Paper presented at the Meeting for the Society of Neuroscience, Los Angeles, CA.

Escobar AL, Monck JR, Fernandez JM, Vergara JL (1994) Localization of the site of $\mathrm{Ca}^{2+}$ release at the level of a single sarcomere in skeletal muscle fibres. Nature 367:739-741.

Escobar AL, Velez P, Kim AM, Cifuentes F, Fill M, Vergara JL (1997) Kinetic properties of DM-nitrophen and calcium indicators: rapid transient response to flash photolysis. Pflügers Arch 434:615-631.

Feller MB, Delaney KR, Tank DW (1996) Presynaptic calcium dynamics at the frog retinotectal synapse. J Neurophysiol 76:381-400.

Fogelson AL, Zucker RS (1985) Presynaptic calcium diffusion from various arrays of single channels. Implications for transmitter release and synaptic facilitation. Biophys J 48:1003-1017.

Gabso M, Neher E, Spira ME (1997) Low mobility of the $\mathrm{Ca}^{2+}$ buffers in axons of cultured Aplysia neurons. Neuron 18:473-481.

Hall JD, Betarbet S, Jaramillo F (1997) Endogenous buffers limit the spread of free calcium in hair cells. Biophys J 73:1243-1252.

Helmchen F, Borst JG, Sakmann B (1997) Calcium dynamics associated with a single action potential in a CNS presynaptic terminal. Biophys $\mathbf{J}$ 72:1458-1471.

Heuser JE, Reese TS (1981) Structural changes after transmitter release at the frog neuromuscular junction. J Cell Biol 88:564-580.

Hiraoka Y, Sedat JW, Agard DA (1990) Determination of threedimensional imaging properties of a light microscope system. Partial confocal behavior in epifluorescence microscopy. Biophys J 57:325-333.

Issa NP, Hudspeth AJ (1996) The entry and clearance of $\mathrm{Ca}^{2+}$ at individual presynaptic active zones of hair cells from the bullfrog's sacculus. Proc Natl Acad Sci USA 93:9527-9532.

Katz B (1969) The release of neural transmitter substances. Liverpool, UK: Liverpool UP. 
Llinas R, Steinberg IZ, Walton K (1981) Relationship between presynaptic calcium current and postsynaptic potential in squid giant synapse. Biophys J 33:323-351.

Markram H, Roth A, Helmchen F (1998) Competitive calcium binding: implications for dendritic calcium signaling. J Comput Neurosci 5:331-348

Mason WT (1993) Fluorescent and luminescent probes for biological activity: a practical guide to technology for quantitative real-time analysis. San Diego: Academic.

Monck JR, Robinson IM, Escobar AL, Vergara JL, Fernandez JM (1994) Pulsed laser imaging of rapid $\mathrm{Ca}^{2+}$ gradients in excitable cells. Biophys J 67:505-514.

Naraghi M (1997) T-jump study of calcium binding kinetics of calcium chelators. Cell Calcium 22:255-268.

Naraghi M, Neher E (1997) Linearized buffered $\mathrm{Ca}^{2+}$ diffusion in microdomains and its implications for calculation of $\left[\mathrm{Ca}^{2+}\right]$ at the mouth of a calcium channel. J Neurosci 17:6961-6973.

Neher E (1998) Vesicle pools and $\mathrm{Ca}^{2+}$ microdomains: new tools for understanding their roles in neurotransmitter release. Neuron 20:389-399.

Obaid AL, Salzberg BM (1996) Micromolar 4-aminopyridine enhances invasion of a vertebrate neurosecretory terminal arborization: optical recording of action potential propagation using an ultrafast photodiode-MOSFET camera and a photodiode array. J Gen Physiol 107:353-368.

Ohana O, Sakmann B (1998) Transmitter release modulation in nerve terminals of rat neocortical pyramidal cells by intracellular calcium buffers. J Physiol (Lond) 513:135-148.

Pape PC, Jong DS, Chandler WK (1995) Calcium release and its voltage dependence in frog cut muscle fibers equilibrated with 20 mM EGTA. J Gen Physiol 106:259-336.

Parnas H, Hovav G, Parnas I (1989) Effect of $\mathrm{Ca}^{2+}$ diffusion on the time course of neurotransmitter release. Biophys J 55:859-874.

Pawley JB (1995) Handbook of biological confocal microscopy. New York: Plenum.

Pumplin DW, Reese TS, Llinas R (1981) Are the presynaptic membrane particles the calcium channels? Proc Natl Acad Sci USA 78:7210-7213.

Regehr WG, Atluri PP (1995) Calcium transients in cerebellar granule cell presynaptic terminals. Biophys J 68:2156-2170.

Roberts WM (1994) Localization of calcium signals by a mobile calcium buffer in frog saccular hair cells. J Neurosci 14:3246-3262.

Roberts WM, Jacobs RA, Hudspeth AJ (1990) Colocalization of ion channels involved in frequency selectivity and synaptic transmission at presynaptic active zones of hair cells. J Neurosci 10:3664-3684.
Sabatini BL, Regehr WG (1996) Timing of neurotransmission at fast synapses in the mammalian brain. Nature 384:170-172.

Sigworth FJ (1980) The variance of sodium current fluctuations at the node of Ranvier. J Physiol (Lond) 307:97-129.

Simon SM, Llinas RR (1985) Compartmentalization of the submembrane calcium activity during calcium influx and its significance in transmitter release. Biophys J 48:485-498.

Sinha SR, Wu LG, Saggau P (1997) Presynaptic calcium dynamics and transmitter release evoked by single action potentials at mammalian central synapses. Biophys J 72:637-651.

Smith PD, Liesegang GW, Berger RL, Czerlinski G, Podolsky RJ (1984) A stopped-flow investigation of calcium ion binding by ethylene glycol bis( $\beta$-aminoethyl ether)- $N, N^{\prime}$-tetraacetic acid. Anal Biochem 143:188-195.

Stockbridge N, Ross WN (1984) Localized $\mathrm{Ca}^{2+}$ and calcium-activated potassium conductances in terminals of a barnacle photoreceptor. Nature 309:266-268.

Sugimori M, Lang EJ, Silver RB, Llinas R (1994) High-resolution measurement of the time course of calcium-concentration microdomains at squid presynaptic terminals. Biol Bull 187:300-303.

Tucker T, Fettiplace R (1995) Confocal imaging of calcium microdomains and calcium extrusion in turtle hair cells. Neuron 15:1323-1335.

Umemiya M, Berger AJ (1995) Single-channel properties of four calcium channel types in rat motoneurons. J Neurosci 15:2218-2224.

Weldon PR, Cohen MW (1979) Development of synaptic ultrastructure at neuromuscular contacts in an amphibian cell culture system. J Neurocytol 8:239-259.

Wilson T (1990) Confocal microscopy. New York: Academic.

$\mathrm{Xu}$ T, Naraghi M, Kang H, Neher E (1997) Kinetic studies of $\mathrm{Ca}^{2+}$ binding and $\mathrm{Ca}^{2+}$ clearance in the cytosol of adrenal chromaffin cells. Biophys J 73:532-545.

Yamada WM, Zucker RS (1992) Time course of transmitter release calculated from simulations of a calcium diffusion model. Biophys $\mathbf{J}$ 61:671-682.

Yazejian B, DiGregorio DA, Vergara JL, Poage RE, Meriney SD, Grinnell AD (1997) Direct measurements of presynaptic calcium and calcium-activated potassium currents regulating neurotransmitter release at cultured Xenopus nerve-muscle synapses. J Neurosci 17:2990-3001.

Zucker RS, Stockbridge N (1983) Presynaptic calcium diffusion and the time courses of transmitter release and synaptic facilitation at the squid giant synapse. J Neurosci 3:1263-1269. 